Herzschr Elektrophys 2013 $\cdot 24: 249-274$ DOI 10.1007/s00399-013-0288-0

Online publiziert: 25. September 2013

(c) Springer-Verlag Berlin Heidelberg 2013
A. Markewitz

Abt. XVII - Herz- und Gefäßchirurgie, Bundeswehrzentralkrankenhaus, Koblenz, Deutschland

\section{Jahresbericht 2011des Deutschen Herzschrittmacher- und Defibrillatorregisters}

\author{
Fachgruppe Herzschrittmacher \\ und AQUA-Institut für angewandte \\ Qualitätsförderung und Forschung \\ im Gesundheitswesen $\mathrm{GmbH}$
}

\section{Inhaltsverzeichnis}

\section{Einleitung}

Datenbasis

Datenvolumen

Demographische Daten

Implantationen

Indikation zur ICD-Implantation

ICD-Systemauswahl

Elektrodenauswahl bei

Implantation

Operationsdaten

Aggregatwechsel

Revisionen/Systemwechsel/

Explantationen

Internationaler Vergleich

Datenbasis

Indikationen zur ICD-Therapie

Operationsdaten

Zusammenfassung und Ausblick

Literatur

Tabellenverzeichnis

Tabellenverzeichnis: Anhang 2

Abbildungsverzeichnis

Anhang 2: Detaillierte Tabellen

Mitglieder der
Bundesfachgruppe
Herzschrittmacher
Für die Bundesärztekammer:
Prof. Dr. S. Behrens, Berlin,
PD Dr. C. Kolb, München
Für die Deutsche Krankenhausgesellschaft:
Prof. Dr. D. W. Behrenbeck, Solingen,
Prof. Dr. G. Fröhlig, Homburg/Saar
Für die Spitzenverbände der Krankenkassen
$\quad$ und den Verband der Privaten
Krankenversicherung:
Dr. S. Knoblich, Recklinghausen,
Dr. J. van Essen, Oberursel
Für die Deutsche Gesellschaft für Kardiologie
$\quad$ - Herz- und Kreislaufforschung:
Prof. Dr. A. Markewitz, Koblenz
Für die Deutsche Gesellschaft für Thorax-,
Herz- und Gefäßchirurgie:
Dr. H. Burger, Bad Nauheim
Für das AQUA-Institut:
Prof. Dr. U. Wiegand, Remscheid
Als Landesvertreter:
Dr. C. Burmeister, Mainz,
Dr. S. Macher-Heidrich, Düsseldorf,
Prof. Dr. B. Nowak, Frankfurt a. M.
Als Patientenvertreter:
W.-D. Trenner, Berlin
Projektteam AQUA-Institut
F. Rüppel
Dr. K. Tasche
Prof. Dr. J. Pauletzki

Teil 2 - Implantierbare Kardioverter-Defibrillatoren (ICD)

Einleitung

Im zweiten Teil des Jahresberichts 2011 werden die Daten zur Therapie mit implantierbaren Kardioverter-Defibrillatoren (ICD) aus dem stationären Bereich in Deutschland vorgestellt und mit den Ergebnissen der Register aus Schweden und der Schweiz [1-3] verglichen.

\section{Datenbasis}

\section{Datenvolumen}

Die Zahl der Krankenhäuser, die in Deutschland ICD-Operationen durchführen, hat in 2011 ebenso zugenommen wie die Zahl an Neuimplantationen. Revisionsoperationen machen erneut fast $20 \%$ der Eingriffe aus $(n=8056 / 43 \cdot 326=18,6 \%)$. Die Rate an Revisionen liegt damit weiterhin deutlich höher als bei den Herzschrittmacheroperationen $(12,8 \%$; $\bullet$ Tab. 1$)$

Die Zahl der Institutionen, die kaum ICD-Operationen durchführen (d. h. weniger als 20 Eingriffe im Berichtsjahr), liegt erneut sehr hoch und deutlich über den vergleichbaren Zahlen bei Herzschrittmacheroperationen. Zusam- 


\begin{tabular}{|c|c|c|}
\hline Datenbasis & 2010 & 2011 \\
\hline \multicolumn{3}{|c|}{$\begin{array}{l}\text { Krankenhäuser (Einrichtungen, die Daten } \\
\text { geliefert haben) }\end{array}$} \\
\hline 09/4: Implantationen & 596 & 648 \\
\hline 09/5: Aggregatwechsel & 481 & 538 \\
\hline $\begin{array}{l}\text { 09/6: Revisionen/System- } \\
\text { wechsel/Explantationen }\end{array}$ & 466 & 511 \\
\hline Alle 3 Leistungsbereiche & 621 & 664 \\
\hline \multicolumn{3}{|l|}{ Eingriffe } \\
\hline 09/4: Implantationen & 25.582 & 28.452 \\
\hline 09/5: Aggregatwechsel & 6002 & 6818 \\
\hline $\begin{array}{l}\text { 09/6: Revisionen/System- } \\
\text { wechsel/Explantationen }\end{array}$ & 7014 & 8056 \\
\hline $\begin{array}{l}\text { Alle } 3 \text { Leistungsbereiche } \\
\text { (Summe) }\end{array}$ & 38.598 & 43.326 \\
\hline
\end{tabular}

Tab. 2 Operationsvolumina der meldenden Krankenhäuser

Anzahl gemeldeter ICD-

20102011 Operationen (Implantationen und Aggregatwechsel)

\begin{tabular}{|lll}
\hline 0 & 7 & 2 \\
\hline $1-19$ & 240 & 249 \\
\hline $20-49$ & 157 & 171 \\
\hline $50-99$ & 121 & 144 \\
\hline $100-199$ & 75 & 72 \\
\hline $200-299$ & 15 & 21 \\
\hline$\geq 300$ & 6 & 5 \\
\hline Summe & 621 & 664 \\
\hline
\end{tabular}

Beispiel: In 171 Krankenhäusern wurden 2011 zwischen 20 und 49 Implantations- oder Aggregatwechseloperationen vorgenommen

Tab. 3 Vollzähligkeit der ausgewerteten Datensätze bzw. Krankenhäuser im Jahr 2010 (Minimaldatensätze berücksichtigt)

\begin{tabular}{|c|c|c|c|}
\hline Datenbasis 2010 & Soll & Ist & Prozent \\
\hline \multicolumn{4}{|l|}{ Krankenhäuser } \\
\hline 09/4: Implantationen & 616 & 596 & 96,8 \\
\hline 09/5: Aggregatwechsel & 502 & $482^{\mathrm{a}}$ & 96,0 \\
\hline 09/6: Revisionen/Systemwechsel/Explantationen & 502 & 466 & 92,8 \\
\hline \multicolumn{4}{|l|}{ Eingriffe } \\
\hline 09/4: Implantationen & 26.984 & $25.598^{\mathrm{b}}$ & 94,9 \\
\hline 09/5: Aggregatwechsel & 6330 & 6005 & 94,9 \\
\hline 09/6: Revisionen/Systemwechsel/Explantationen & 7696 & 7077 & 92,0 \\
\hline \multicolumn{4}{|c|}{$\begin{array}{l}\text { aDie Angaben zur Anzahl der meldenden Krankenhäuser weichen von den Einträgen in } \mathbf{\bullet} \text { Tab. } 1 \text { ab, da hier } \\
\text { auch die Minimaldatensätze mitgezählt werden } \\
\text { bDie Angaben zu den vorhandenen Datensätzen weichen von den Einträgen in } \bullet \text { Tab. } 1 \text { ab, da hier auch } \\
\text { die Minimaldatensätze mitgezählt werden. Gleiches gilt für die Leistungsbereiche 09/5 und 09/6 }\end{array}$} \\
\hline
\end{tabular}

Tab. 4 Vollzähligkeit der ausgewerteten Datensätze bzw. Krankenhäuser im Jahr 2011 (Minimaldatensätze berücksichtigt)

\begin{tabular}{llll}
\hline Datenbasis 2011 & Soll & Ist & Prozent \\
\hline Krankenhäuser & & & \\
\hline 09/4: Implantationen & 651 & $641^{\text {a }}$ & 98,5 \\
\hline 09/5: Aggregatwechsel & 550 & 533 & 96,9 \\
\hline 09/6: Revisionen/Systemwechsel/Explantationen & 520 & 506 & 97,3 \\
\hline Eingriffe & & & \\
\hline 09/4: Implantationen & 28.957 & $28.497^{\mathrm{b}}$ & 98,4 \\
\hline 09/5: Aggregatwechsel & 6927 & 6833 & 98,6 \\
\hline 09/6: Revisionen/Systemwechsel/Explantationen & 8360 & 8110 & 97,0 \\
\hline
\end{tabular}

aHier weichen die Angaben von den Einträgen in $\mathbf{0}$ Tab. 1 ab, da letztere auf Pseudonymen basieren, welche der Bundesauswertungsstelle (AQUA-Institut) von den Landesgeschäftsstellen für Qualitätssicherung (LQS) übermittelt werden - dabei werden in einigen Fällen für mehrere Betriebsstätten eines Krankenhauses unterschiedliche Pseudonyme übermittelt. Gleiches gilt für die Leistungsbereiche 09/5 und 09/6 bSiehe Fußnote 2 in $\bullet$ Tab. 3

mengefasst gab es wie im Vorjahr in zwei Drittel der Krankenhäuser weniger als 1 ICD-Operation pro Woche (• Tab. 2, - Abb. 1, Anhang 2 Tab. 1). Die neu eingeführte Kategorie „0“ in $\bullet$ Tab. 2 be- schreibt die für den Praktiker zumindest erstaunliche Tatsache, dass es sowohl in 2010 als auch in 2011 Häuser gab, die ausschließlich Revisionen, Systemwechsel oder Explantationen durchgeführt ha- ben. Man kann hier nur spekulieren, dass es sich um Institutionen handelt, die ICDs explantiert haben, z. B. im Rahmen einer Herztransplantation, ansonsten aber die operative Therapie bei Patienten mit kardialen Rhythmusimplantaten an andere abgegeben haben.

Die Datenvollständigkeit (• Tab. 4) hat sich im Vergleich zum Vorjahr erwartungsgemäß deutlich gebessert (• Tab. 3 ) und liegt bereits im 2. Verfahrensjahr im Bereich der Zahlen bei den Herzschrittmachern (• Tab. 4 in Teil 1 des Berichts).

\section{Demographische Daten}

Wie im Vorjahr liegt die Zahl der Eingriffe pro Institution bei den Neuimplantationen und Aggregatwechseln erwartungsgemäß niedriger als für die Herzschrittmacher beobachtet, bei den Revisionen jedoch mit beunruhigend steigender Tendenz über den Zahlen der Herzschrittmacheroperationen. Dabei sind vergleichsweise deutlich mehr Männer vertreten, und die ICD-Patienten bleiben im Durchschnitt deutlich jünger als die Schrittmacherpatienten (• Tab. 5 und Anhang 2 Tab. 2). Die Frage der Verweildauer und deren Implikationen für die Vergütung wurde an dieser Stelle sowohl für die Herzschrittmachereingriffe als auch die ICD-Operationen oft genug gestellt und bedarf keiner Wiederholung. Es ist allerdings bedauerlich, dass die Registerzahlen offensichtlich kaum oder gar nicht zur Kenntnis genommen werden, insbesondere wenn man bedenkt, mit welcher Konstanz ansonsten weitaus weniger gut fundierte Zahlen zur Befeuerung gesundheitsökonomischer Debatten ins Feld geführt werden.

\section{Implantationen}

\section{Indikation zur ICD-Implantation}

Bei den Indikationen zur ICD-Implantation überwiegt weiterhin die Primärprävention (• Tab. 6), wobei die nicht unbeträchtliche Spannweite der Ergebnisse auffällt (• Tab. 7 und $\bullet$ Abb. 2). Allerdings nimmt die Zahl der Häuser ab, die in weniger als $20 \%$ eine primärprophylaktische ICD-Implantation durchführ- 
Tab. 5 Demographische Daten zu Implantationen, Aggregatwechseln und Revisionen/ Systemwechseln/Explantationen

\begin{tabular}{lll} 
& 2010 & 2011 \\
\hline 09/4 Implantationen & 25.582 & $\mathbf{2 8 . 4 5 2}$ \\
\hline Im Mittel je Institution & 42,9 & 43,9 \\
\hline Geschlecht & & \\
\hline - Männlich (\%) & 79,8 & 78,6 \\
\hline - Weiblich (\%) & 20,2 & 21,4 \\
\hline Mittleres Alter (Jahre) & & \\
\hline - Bei Männern & 66,0 & 66,3 \\
\hline - Bei Frauen & 65,6 & 66,5 \\
\hline Patienten <60 Jahre (\%) & 26,3 & 25,5 \\
\hline Permanente atriale oder ventrikuläre Stimulationsbedürftigkeit (\%) & 38,1 & 39,0 \\
\hline Mittlere postoperative Verweildauer (Tage) & 4,9 & 4,7 \\
\hline 09/5 Aggregatwechsel & 6002 & 6818 \\
\hline Im Mittel je Institution & 12,5 & 12,7 \\
\hline Mittleres Alter (Jahre) & & \\
\hline - Bei Männern & 67,9 & 68,2 \\
\hline - Bei Frauen & 66,7 & 67,5 \\
\hline Mittlere Zeit zw. Implantation und Austausch (Jahre) & 5,5 & 5,7 \\
\hline Mittlere postoperative Verweildauer (Tage) & 2,7 & 2,7 \\
\hline 09/6 Revisionen/Systemwechsel/Explantationen & 7014 & 8056 \\
\hline Im Mittel je Institution & 15,1 & 15,8 \\
\hline Geschlecht & 66,1 & 66,4 \\
\hline - Männlich (\%) & 63,7 & 64,4 \\
\hline - Weiblich (\%) & 6,2 & 5,9 \\
\hline Mittleres Alter (Jahre) & 20,2 & 21,3 \\
\hline - Bei Männern & & \\
\hline - Bei Frauen & & \\
\hline Mittlere postoperative Verweildauer (Tage) & & \\
\hline & & \\
\hline
\end{tabular}

\begin{tabular}{|lllll}
\hline Tab. 6 Führende Indikation der ICD-Implantation & & & \\
\hline Führende Indikation für ICD-Implantation & $\mathbf{2 0 1 0}$ & & $\mathbf{2 0 1 1}$ & \\
\hline & $\boldsymbol{n}$ & Prozent & $\boldsymbol{n}$ & Prozent \\
\hline Primärprävention & 17.936 & 70,1 & 20.450 & 71,9 \\
\hline Sekundärprävention & 7646 & 29,9 & 8002 & 28,1 \\
\hline Summe & 25.582 & 100,0 & 28.452 & 100,0 \\
\hline
\end{tabular}

Tab. 7 Verteilung der Indikation

Primärprävention bei Implantationen in den einzelnen Krankenhäusern

\begin{tabular}{lll} 
Verteilung & $\mathbf{2 0 1 0}$ & $\mathbf{2 0 1 1}$ \\
\hline$<10$ & $n$ & $n$ \\
\hline 10 bis $<20$ & 5 & 22 \\
\hline 20 bis $<30$ & 7 & 15 \\
\hline 30 bis $<40$ & 10 & 19 \\
\hline 40 bis $<50$ & 33 & 26 \\
\hline 50 bis $<60$ & 91 & 77 \\
\hline 60 bis $<70$ & 125 & 127 \\
\hline 70 bis $<80$ & 144 & 146 \\
\hline 80 bis $<90$ & 81 & 116 \\
\hline$\geq 90$ & 71 & 99 \\
\hline Summe & 596 & 648 \\
\hline
\end{tabular}

ten, bleibt aber ohne weitere Informationen weiterhin nicht zu erklären.

Die häufigsten EKG-Befunde bzw. Symptome, die eine sekundärpräventive ICD-Implantation begründeten, sind in - Abb. 3 aufgeführt. Weitere Details zu den indikationsbegründenden Ereignissen bzw. Symptomen sind in Anhang 2

- Tab. 3 und $4 \mathrm{zu}$ finden.

Wie erwartet, hat sich das Ausmaß der Leitlinienkonformität im Vergleich zum Vorjahr deutlich verbessert (Anhang 2 - Tab. 5). Interessierte finden im Anhang 2 nähere Informationen zur KHK und anderen Ätiologien sowie zur medikamentösen Herzinsuffizienztherapie bei den $\mathrm{Pa}$ tienten, die sich einer ICD-Implantation unterzogen (Anhang 2 Tab. 6, 7, 8 und 10). Im Vergleich zum Vorjahr haben sich diese Zahlen nur wenig verändert.

Wer sich für die bradykarden Rhythmusstörungen der ICD-Patienten interessiert, wird in Anhang 2 Tab. 11 Antworten auf seine Fragen finden.

\section{ICD-Systemauswahl}

Bei der Auswahl der ICD-Systeme zeigt sich im Vergleich zum Vorjahr ein nahezu unverändertes Bild (• Abb. 4): VVIICDs werden am häufigsten implantiert, und die CRT-Systeme werden häufiger als die DDD-ICD eingesetzt, wobei tendenziell die CRT-Systeme zunehmen und die VVI-Systeme abnehmen.

\section{Elektrodenauswahl bei Implantation}

Bei der Auswahl der rechtsventrikulären Defibrillationssonde fällt erneut, allerdings mit abnehmender Tendenz, die für den Praktiker nicht vollständig nachvollziehbare, häufige Verwendung einer Dual-coil-Elektrode auf (• Tab. 8). Auf die fehlende Evidenz für einen Vorteil gegenüber der Single-coil-Elektrode [4] und die erfahrungsgemäß schlechtere Entfernbarkeit der Dual-coil-Sonden wurde bereits im Vorjahr hingewiesen.

Die aus Sicht des Praktikers zumindest sehr erwägenswerte Alternative zur Dual-coil-Sonde, die Implantation einer 2. Defibrillationselektrode, wenn eine Single-coil-Elektrode nicht ausreicht, wird weiterhin nur sehr selten durchgeführt (• Tab.9).

\section{Operationsdaten}

Wie im Vorjahr nimmt der Praktiker mit Überraschung zur Kenntnis, dass die Vena cephalica noch viel seltener als bei der Schrittmacherimplantation verwendet wird (- Tab. 10), obwohl dies bei der Patientengruppe, die einen ICD benötigen, erfahrungsgemäß häufiger möglich ist. Dabei hat die Zahl der Häuser, in denen die Vena cephalica in weniger als $10 \%$ der Fälle verwendet wird auf 258 zugenommen, aber auch die Zahl der Häuser, in denen es in mehr als $60 \%$ der Fälle gelingt, dies zu tun, ist größer geworden $(n=210$ 


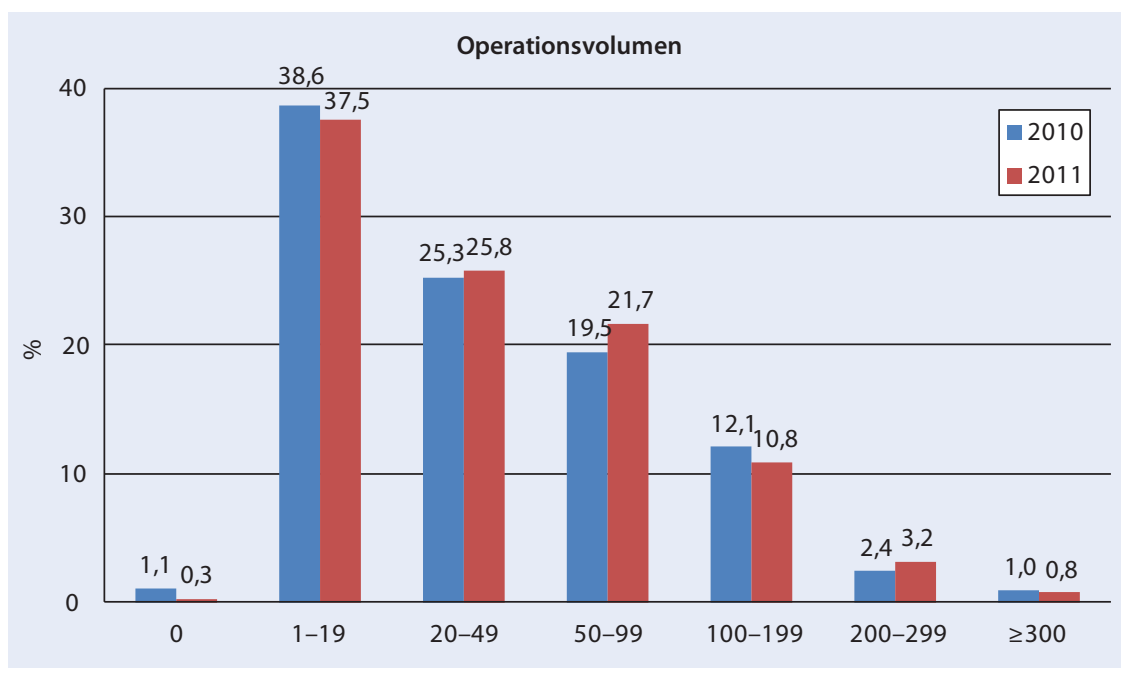

Abb. 1 A Prozentuale Verteilung des Operationsvolumens der einzelnen Krankenhäuser (Beispiel: Im Jahr 2011 führten 25,8\% der Einrichtungen zwischen 20 und 49 ICD-Implantationen bzw.-Aggregatwechsel durch, im Jahr 2010 waren es $25,3 \%$ )

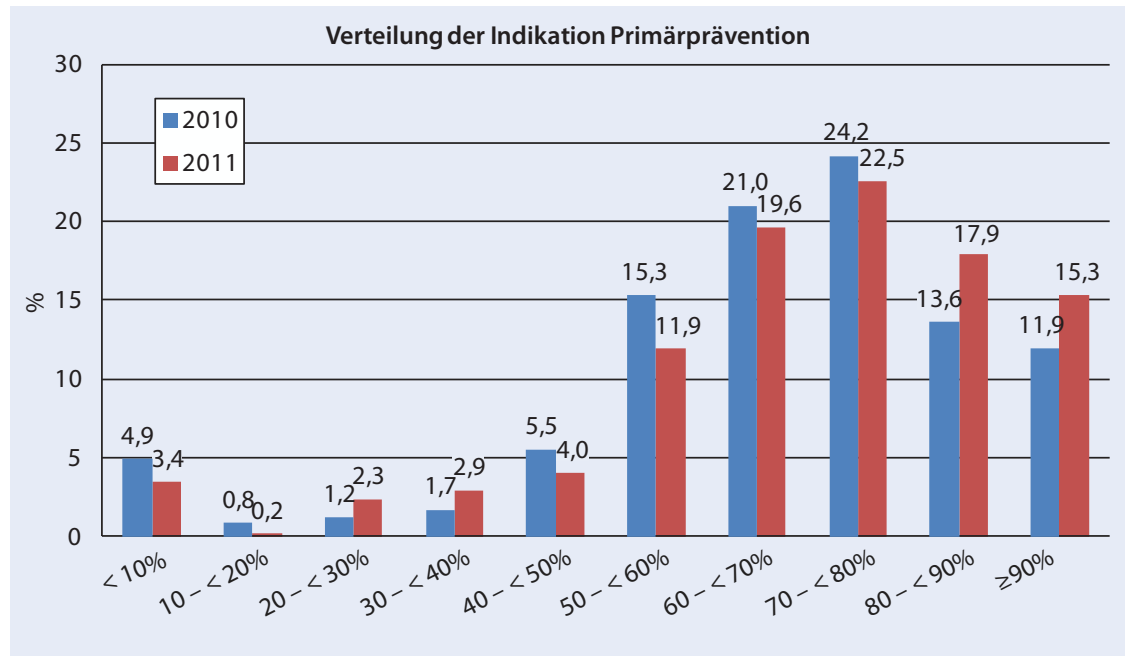

Abb. $2 \Delta$ Prozentuale Verteilung der Indikation Primärprävention in den einzelnen Krankenhäusern (Beispiel: Bei 4,0\% der Krankenhäuser lag der Anteil der Indikation Primärprävention zwischen 40 und $<50 \%$ in 2011)

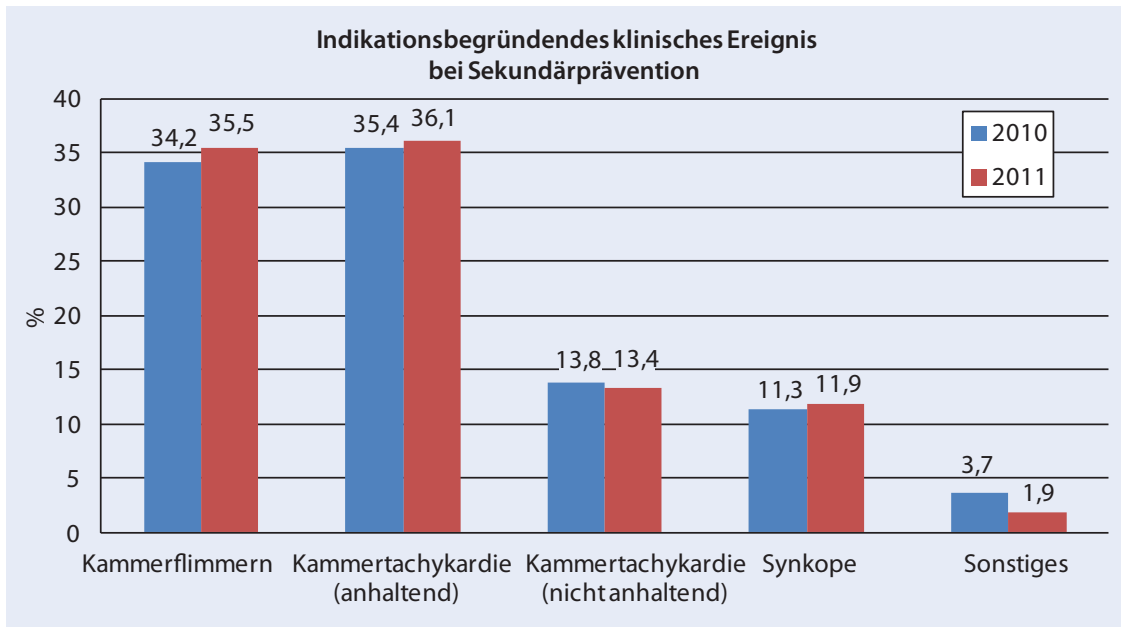

Abb. $3 \Delta$ Prozentuale Verteilung der indikationsbegründenden klinischen Ereignisse bei Sekundärprävention vs. $n=192$ in 2010; $\bullet$ Abb. 5). Dennoch werden weiterhin die entsprechenden Vergleichszahlen aus der Herzschrittmachertherapie deutlich über- bzw. unterboten.

Die Operationszeiten bei der ICD-Implantation bleiben wie im Vorjahr über denen bei den Herzschrittmachereingriffen (• Tab. 11), allerdings mit erkennbarer Tendenz zu kürzeren Implantationszeiten sowohl bei den einzelnen Eingriffen als auch bei den Krankenhäusern (- Abb. 6, 7, 8, 9, 10 und 11 und Anhang 2 - Tab. 11). Die Durchleuchtungszeiten sind weiter fast identisch (• Tab. 12).

Der im Vorjahr nur bedingt erkennbare Zusammenhang zwischen Operationszeiten und Fallzahlen bestätigt sich ähnlich wie bei den HerzschrittmacherImplantationen in 2011 für alle Fallzahlklassen auch bei den ICD-Implantationen (- Tab. 13).

Bei den Ergebnissen der Reizschwellenbestimmung sowie der Ermittlung der intrakardialen Signalamplituden hat sich im Vergleich zum Vorjahr nichts geändert. Sie sind überdies weiterhin nahezu identisch mit den Ergebnissen der Herzschrittmachertherapie (• Tab. 14).

Details der Positionen und der Ergebnisse der intraoperativen Messungen der üblicherweise für die CRT-Stimulation verwendeten 2. und/oder 3. Ventrikelsonde findet der Interessierte in Anhang 2

- Tab. 12, 13 und 14.

Bei den Ergebnissen für die Bestimmung der sog. Defibrillationsschwelle (DFT) fällt auf, dass sie zunehmend weniger durchgeführt wird (• Tab. 15), obwohl bislang unklar ist, ob ein Verzicht darauf vertretbar oder gar sinnvoll ist $[5,6]$.

Der Sicherheitsabstand von $>10$ J zwischen DFT und maximaler Energie des implantierten ICD-Aggregats konnte erneut nicht bei allen Patienten erreicht werden, wobei die prognostische Bedeutung dieses Sicherheitsabstands ebenfalls noch nicht endgültig geklärt ist [5].

In Kenntnis der Ergebnisse neuerer retrospektiver Erhebungen stellt sich dem Verfasser erneut die Frage, ob es sinnvoll und/oder vertretbar ist, bei der Implantation eines Systems auf die Überprüfung der Funktion zu verzichten, von der im weiteren Verlauf das Leben der Patienten abhängt. Die Diskussion um dieses Thema bleibt somit spannend. 
Tab. 8 Defibrillationselektroden und Position bei Implantationen der ersten Ventrikelsonde/ Defibrillationssonde

\begin{tabular}{lllll} 
& 2010 & & 2011 & \\
\hline Defibrillationselektroden & $n$ & Prozent & $n$ & Prozent \\
\hline Single coil & & & & \\
\hline Dual coil & 9896 & 38,7 & 11.935 & 41,9 \\
\hline Position & 15.686 & 61,3 & 16.517 & 58,1 \\
\hline Rechtsventrikulärer Apex & & & & \\
\hline Rechtsventrikuläres Septum & 22.786 & 89,1 & 24.813 & 87,2 \\
\hline Andere & 2557 & 10,0 & 3421 & 12,0 \\
\hline
\end{tabular}

Tab. 9 Weitere Defibrillationssonden

\begin{tabular}{lllll} 
Art und/oder Implantationsort & $\mathbf{2 0 1 0}$ & & $\mathbf{2 0 1 1}$ & \\
\hline Vena cava superior & $\boldsymbol{n}$ & Prozent & $\boldsymbol{n}$ & Prozent \\
\hline Vena subclavia & 433 & 1,69 & 429 & 1,51 \\
\hline Rechter Vorhof & 125 & 0,49 & 99 & 0,35 \\
\hline Subkutan (Sub-Q-Array) & 57 & 0,22 & 50 & 0,18 \\
\hline Epimyokardial (Patch-Elektrode) & 16 & 0,06 & 18 & 0,06 \\
\hline Mehrere & 5 & 0,02 & 7 & 0,02 \\
\hline Andere & 2 & 0,01 & 0 & 0,00 \\
\hline Summe: alle weiteren Defibrillationssonden & 696 & 2,72 & 707 & 2,48 \\
\hline Keine weitere(n) Defibrillationssonde(n) & 24.886 & 97,28 & 27.745 & 97,52 \\
\hline
\end{tabular}

Tab. 10 Venöser Zugang bei ICD-Implantationen

\begin{tabular}{|lllll}
\hline Venöser Zugang & $\mathbf{2 0 1 0}$ & & $\mathbf{2 0 1 1}$ & \\
\hline & $\boldsymbol{n}$ & Prozent & $\boldsymbol{n}$ & Prozent \\
\hline Vena cephalica & 8803 & 34,4 & 9646 & 33,9 \\
\hline Vena subclavia & 18.829 & 73,6 & 21.191 & 74,5 \\
\hline Andere & 681 & 2,7 & 644 & 2,3 \\
\hline
\end{tabular}

Tab. 11 Operationszeiten in Minuten bei Implantationen 2010 und 2011 (bezogen auf alle Fälle mit gültiger Angabe zur Operationsdauer $>0$ )

\begin{tabular}{lllllll} 
ICD-System & $\mathbf{2 0 1 0}$ & $\mathbf{2 0 1 1}$ & & & & \\
\hline & MW & $\boldsymbol{n}$ & MW & SD & Median & $\mathbf{7 5 \%}$ \\
\hline VVI & 52,6 & 12.133 & 49,3 & 24,6 & 45,0 & 60,0 \\
\hline DDD & 74,0 & 7434 & 70,2 & 37,5 & 60,0 & 82,0 \\
\hline CRT & 125,4 & 8519 & 120,8 & 51,5 & 114,0 & 150,0 \\
\hline Sonstige (inkl. VDD) & 82,6 & 366 & 71,4 & 51,1 & 55,0 & 80,0 \\
\hline Summe & 79,1 & 28.452 & 76,5 & 48,6 & 60,0 & 97,0 \\
\hline
\end{tabular}

MW Mittelwert, SD Standardabweichung

Tab. 12 Durchleuchtungszeiten in Minuten bei Implantationen 2010 und 2011 (bezogen auf alle Fälle mit gültiger Angabe zur Durchleuchtungsdauer $>0$ )

\begin{tabular}{lllllll}
\hline ICD-System & $\mathbf{2 0 1 0}$ & $\mathbf{2 0 1 1}$ & & & & \\
\hline & $\mathbf{M W}$ & $\boldsymbol{n}$ & $\mathbf{M W}$ & SD & Median & $\mathbf{7 5 \%}$ \\
\hline VVI & 4,0 & 11.986 & 3,8 & 5,0 & 2,4 & 4,3 \\
\hline DDD & 8,0 & 7346 & 7,3 & 9,9 & 4,4 & 8,0 \\
\hline CRT & 22,1 & 8403 & 21,3 & 17,0 & 16,3 & 27,4 \\
\hline Sonstige (inkl. VDD) & 14,3 & 337 & 8,2 & 14,9 & 2,9 & 6,0 \\
\hline Summe & 10,2 & 28.072 & 10,0 & 13,5 & 4,8 & 12,0 \\
\hline MW Mittelwert, SD Standardabweichung & & & & \\
\hline
\end{tabular}

Die Angaben zur Aggregatposition zeigen erneut 2 Befunde, die zumindest vom Verfasser so nicht erwartet wurden (• Tab. 16): Zum einen werden weiterhin weniger als die Hälfte der Aggregate submuskulär implantiert, was im Hinblick auf die nach wie vor nicht kleinen Dimensionen mancher Aggregate nicht bei jedem Patienten unbedenklich erscheint. Zum zweiten sei erneut der Hinweis gestattet, dass die sog. subkutane Tasche lege artis unter der Faszie des M. pectoralis major, also subfaszial angelegt wird. Es bleibt im Sinne der Patienten zu hoffen; dass nicht tatsächlich 23,2\% der Aggregattaschen irgendwo im bisweilen ausgeprägt vorhandenen subkutanen Fettgewebe angelegt wurden. Eigene Erfahrungen bei Revisionen wegen einer schmerzhaften Aggregattasche lassen allerdings befürchten, dass diese Hoffnung trügt.

Die Häufigkeit perioperativer Komplikationen hat in allen Kategorien bis auf die Pneumothraces abgenommen (- Abb. 12 und 0 Tab. 17), wobei die Zunahme der Penumothoraces nicht alleine durch die zunehmende Beliebtheit der Vena subclavia als Zugangsweg erklärbar ist.

Im Vergleich zu den Schrittmacherimplantationen sind Sondendislokationen und die Gruppe der „sonstigen“ Komplikationen seltener, alle anderen Komplikationen jedoch häufiger zu beobachten.

Daher ist auch die Zahl der Krankenhäuser, in denen die Vorhofsonde in weniger als $1 \%$ disloziert, weiterhin um über $20 \%$ höher als bei den Schrittmachern, und die Zahl an Krankenhäusern, in denen die Vorhofsonde in mehr als 5\% disloziert, mit 3,7\% zwar vergleichsweise angestiegen, aber immer noch niedriger als bei den Schrittmacherimplantationen (8,8\%; • Abb. 13).

Wie im Vorjahr findet sich auch in 2011 ein eindeutiger Zusammenhang zwischen der Gesamtkomplikationsrate und dem venösen Zugangsweg (• Tab. 18). Der Zusammenhang zwischen Fallzahlen und Komplikationen zeigt erneut Unterschiede zu den entsprechenden Zahlen bei Schrittmacherimplantation. Der Zusammenhang existiert nämlich nur für den Zugang über die Vena cephalica und ist dort bedingt durch die niedrigere Rate an Taschenhämatomen und Son- 


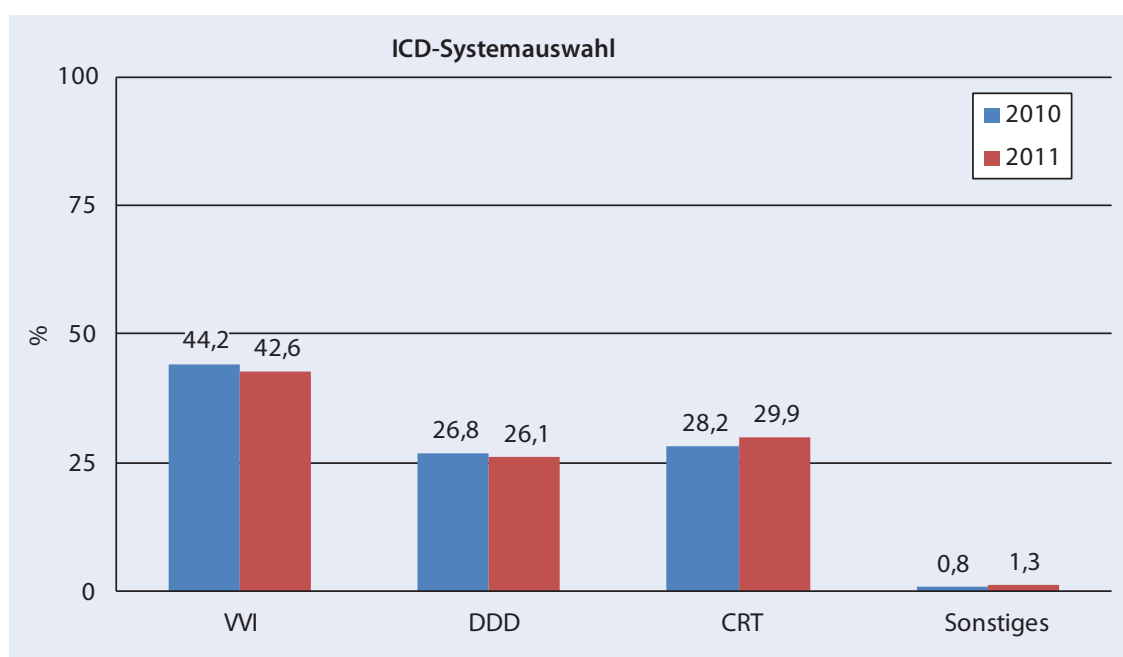

Abb. 4 \ Prozentuale Verteilung der ICD-Systeme bei Implantationen (Sonstiges Sonstige + VDD)

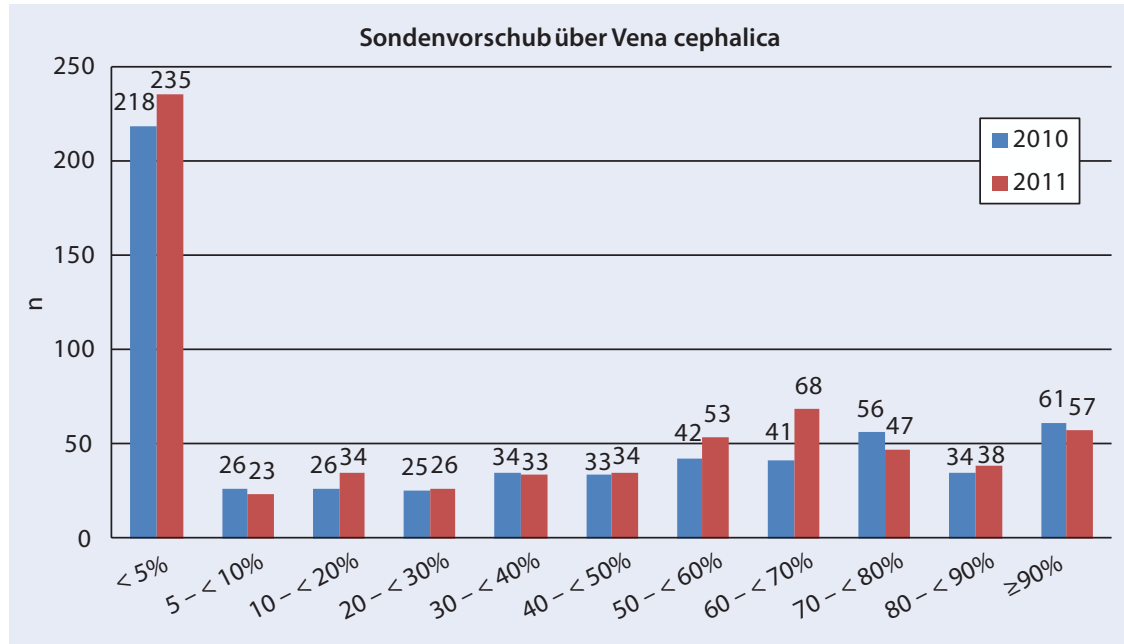

Abb. 5 \ Verteilung der Häufigkeit der Verwendung der Vena cephalica bei der Implantation (Beispiel: Bei 26 Krankenhäusern lag der Anteil der Patienten mit Verwendung der Vena cephalica zwischen 20 und $<30 \%$ in 2011)

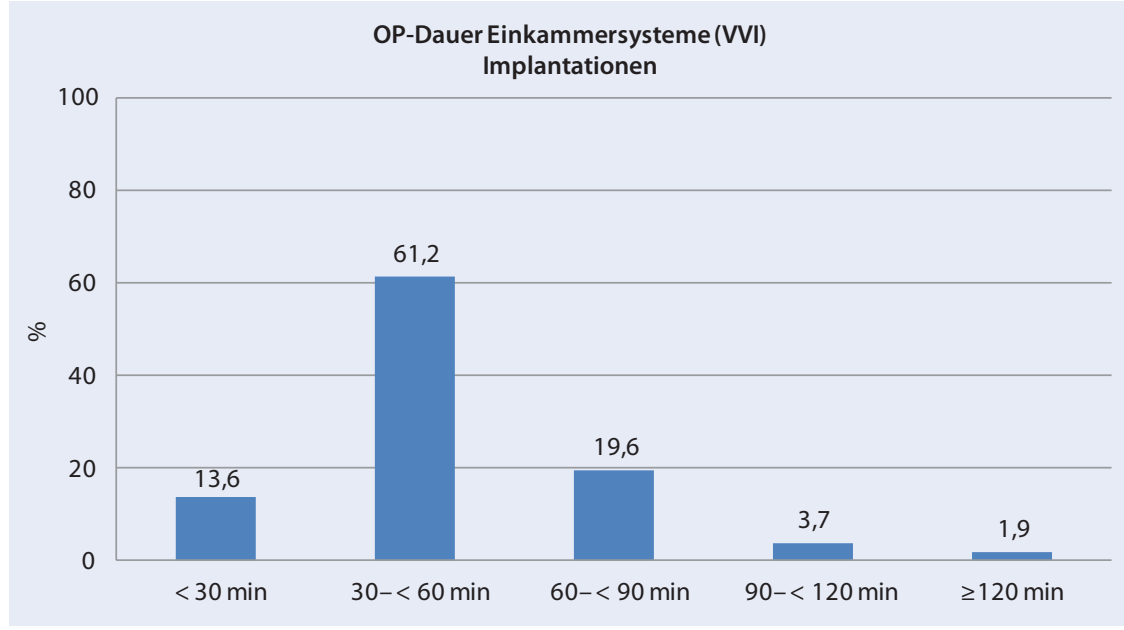

Abb. $6 \Delta$ Prozentuale Verteilung der Operationszeiten bei der Implantation von Einkammersystemen (VVI) bezogen auf alle Implantationen von Einkammersystemen (VVl; Beispiel: Bei 61,2\% der implantierten Einkammersysteme lag die Operationsdauer zwischen 30 und $<60 \mathrm{~min}$ ) dendislokationen in den Fallzahlklassen mit mehr als einem Eingriff pro Woche (• Tab. 19). Im Gegensatz dazu ist in der Subklavia-Gruppe die Komplikationsrate in der Fallzahlklasse $>100$ erneut höher als in der Fallzahlklasse 51-100, aber erstmals niedriger als in der Fallzahlklasse bis 50. Es entspricht sowohl der praktischen Erfahrung als auch den Zahlen dieses Registers, wenn beim Vergleich der beiden Zugangswege die Vena cephalica in jeder Fallzahlklasse deutlich besser abschneidet.

\section{Aggregatwechsel}

Die relative Häufigkeit dieser Eingriffe entspricht mit $15,7 \%$ denen bei der Schrittmachertherapie (15,8\%). Die Laufzeiten der Aggregate sind allerdings weiterhin erheblich kürzer $(\bullet$ Abb. 14). Wie im Vorjahr mit Bedauern erwähnt, stehen Informationen zu Laufzeitunterschieden je nach Hersteller frühestens ab 2013 zur Verfügung.

Wie erwartet und ohne größere Unterschiede zu den Ergebnissen des Vorjahres, halten VVI-ICD-Aggregate länger als DDD-Aggregate und diese wiederum länger als CRT-ICD-Aggregate (• Tab. 20).

Etwas mehr als ein Drittel der Aggregate hatte zum Zeitpunkt des Austauschs Therapien abgegeben, die überwiegend als adäquat eingestuft wurden. Die Angabe von lediglich $4,4 \%$ inadäquater Therapieabgaben (- Tab. 21) erscheint nach den Ergebnissen der Literatur weiterhin zu niedrig $[7,8]$.

Die Rate an durchgeführten DFT-Testungen lag für die Austauschoperationen erneut deutlich niedriger als bei den Neuimplantationen und nimmt im Vergleich zum Vorjahr weiter ab (• Tab. 22).

\section{Revisionen/Systemwechsel/ Explantationen}

Wie eingangs erwähnt, ist die relative Häufigkeit dieser Eingriffe (im Folgenden als Revisionen zusammengefasst) bei den ICD mit 18,6\% deutlich höher als bei Herzschrittmachern.

Wie bei den Herzschrittmachern sind Revisionen für andere Häuser deutlich seltener als Revisionen bei Patienten, die zuvor am eigenen Hause operiert wurden, 
Tab. 13 Mittlere Operationsdauer der Systeme nach Fallzahlklassen (Anzahl an ICD-Implantationen pro Krankenhaus in 2011)

\begin{tabular}{|lllll}
\hline OP-Dauer & \multicolumn{2}{l}{ Fallzahlklassen } & \\
& $\mathbf{5 0}$ & $\mathbf{5 1 - 1 0 0}$ & $>\mathbf{1 0 0}$ & Gesamt \\
\hline Einkammersysteme (VVI) & & & & \\
\hline Anzahl ( $n$ ) & 3846 & 4007 & 4280 & 12.133 \\
\hline Mittelwert (min) & 53,4 & 49,0 & 45,8 & 49,3 \\
\hline Zweikammersysteme (VDD, DDD) & & & & \\
\hline Anzahl (n) & 2469 & 2725 & 2467 & 7661 \\
\hline Mittelwert (min) & 74,1 & 69,1 & 66,3 & 69,8 \\
\hline CRT-Systeme & & & & \\
\hline Anzahl (n) & 1812 & 2873 & 3834 & 8519 \\
\hline Mittelwert (min) & 138,3 & 123,8 & 110,4 & 120,8 \\
\hline
\end{tabular}

Tab. 14 Ergebnisse der Reizschwellenmessungen und Bestimmungen der intrakardialen Signalamplituden bei Implantationen (jeweils bezogen auf alle Fälle mit gültiger Angabe)

\begin{tabular}{|c|c|c|c|c|}
\hline & Anzahl (n) & MW & SD & Median \\
\hline \multicolumn{5}{|l|}{ Vorhof (1. Sonde) } \\
\hline Reizschwelle & 13.745 & $0,8 \mathrm{~V}$ & $0,5 \mathrm{~V}$ & $0,7 \mathrm{~V}$ \\
\hline P-Wellen-Amplitude & 15.249 & $3,0 \mathrm{mV}$ & $1,8 \mathrm{mV}$ & $2,7 \mathrm{mV}$ \\
\hline \multicolumn{5}{|l|}{ Ventrikel (1. Sonde) ${ }^{a}$} \\
\hline Reizschwelle & 28.267 & $0,7 \mathrm{~V}$ & $0,4 \mathrm{~V}$ & $0,6 \mathrm{~V}$ \\
\hline R-Amplitude & 27.918 & $13,6 \mathrm{mV}$ & $5,5 \mathrm{mV}$ & $12,0 \mathrm{mV}$ \\
\hline
\end{tabular}

Tab. 15 Durchführung des intraoperativen Defibrillationstests (Implantationen)

\begin{tabular}{|lllll|}
\hline Intraoperativer Defibrillationstest bei & $\mathbf{2 0 1 0}$ & & $\mathbf{2 0 1 1}$ \\
\cline { 2 - 6 } Implantationen durchgeführt? & $\boldsymbol{n}$ & Prozent & $\boldsymbol{n}$ & Prozent \\
\hline Ja & 16.862 & 65,9 & 15.668 & 55,1 \\
\hline Sicherheitsabstand: nein & 491 & 1,9 & 487 & 1,7 \\
\hline Sicherheitsabstand: ja & 16.371 & 64,0 & 15.181 & 53,4 \\
\hline Nein, wegen intrakardialen Thromben & 1536 & 6,0 & 1871 & 6,6 \\
\hline $\begin{array}{l}\text { Nein, wegen hämodynamischer Instabilität (kate- } \\
\text { cholaminpflichtig oder Lungenödem) }\end{array}$ & 826 & 3,2 & 1231 & 4,3 \\
\hline Nein, aus sonstigen Gründen & 6358 & 24,9 & 9682 & 34,0 \\
\hline
\end{tabular}

Tab. 16 Position der Aggregattasche

\begin{tabular}{|lllll|}
\hline Aggregatposition & $\mathbf{2 0 1 0}$ & & $\mathbf{2 0 1 1}$ & \\
\hline & $\boldsymbol{n}$ & Prozent & $\boldsymbol{n}$ & Prozent \\
\hline Infraklavikulär subkutan & 6265 & 24,5 & 6593 & 23,2 \\
\hline Infraklavikulär subfaszial & 6677 & 26,1 & 8084 & 28,4 \\
\hline Infraklavikulär submuskulär & 12.555 & 49,1 & 13.680 & 48,1 \\
\hline Abdominal & 17 & 0,1 & 16 & 0,1 \\
\hline Andere & 68 & 0,3 & 79 & 0,3 \\
\hline
\end{tabular}

allerdings mit leicht zunehmender Ten$\operatorname{denz}(-T a b .23)$. Sondenprobleme stellen weiter die häufigste Indikation zur Revision dar (• Tab. 24).

Bei den ICD-Aggregat-Problemen und Systemumwandlungen sind kaum Veränderungen zum Vorjahr zu verzeichnen (• Tab. 25).
Bei den Sondenproblemen (• Tab. 26) sind die rechtsventrikulären ICD-Sonden weiterhin am häufigsten betroffen. Wie im Vorjahr sind die Kennzahlen für die relative Häufigkeit einer Dislokation und eines Stimulationsverlusts/Reizschwellenanstiegs deutlich niedriger als bei den Herzschrittmacher-Revisionen.
Im Gegensatz dazu sind Sondenbrüche/ Isolationsdefekte, Oversensing und Infektionen z. T. deutlich häufiger. Auf die zahlenmäßig derzeit nicht abschätzbare Problematik der konstruktionsbedingten Sondendysfunktionen wie Sondenbrüche und Isolationsdefekte wurde schon im Vorjahr hingewiesen $[9,10]$. Die höhere Zahl an Infektionen bleibt weiterhin unklar.Bei der chirurgischen Korrektur von Sondenproblemen ( Abb. 15 und 16 und Anhang 2 Tab. 15) bleibt es wie in 2010 dabei, dass im Vergleich zu den Sondenrevisionen bei Herzschrittmachern Sonden bei ICD-Patienten sowohl im Vorhof als auch im Ventrikel häufiger explantiert und weniger stillgelegt werden.

Wie bei allen anderen Eingriffen wird auch bei Revisionsoperationen die DFT immer seltener ermittelt (Anhang 2 - Tab. 16), was zumindest zum Teil dadurch erklärt werden kann, dass eine DFT-Testung auch nicht bei jeder Revision notwendig erscheint, solange die RV-Elektrode nicht verändert wird und die Aggregatposition unverändert bleibt.

Komplikationen nach Austauschoperationen oder Revisionsoperationen treten weiterhin häufiger auf als bei den entsprechenden Schrittmachereingriffen (- Tab. 27). Dies ist bei den Austauschoperationen bedingt durch die vergleichsweise häufigeren Taschenhämatome und bei den Revisionseingriffen v. a. durch die höhere Rate von Pneumo- und Hämatothoraces und Infektionen, wohingegen Sondenprobleme seltener beobachtet wurden.

Todesfälle im Zusammenhang mit ICD-Operationen ( $\bullet$ Tab. 28) traten bei den Neuimplantationen seltener auf als bei den Schrittmacherimplantationen (0,6 vs. $1,3 \%)$. Die Rate bei den Austauschoperationen liegt etwas niedriger ( 0,2 vs. $0,3 \%$ ) und bei den Revisionsoperationen etwas höher (1,3 vs. $1,2 \%)$.

\section{Internationaler Vergleich}

\section{Datenbasis}

Für einen Vergleich der Daten aus Deutschland mit anderen europäischen Registerberichten liegen für das Jahr 2011 erneut die Berichte aus der Schweiz und Schweden vor [2,3]. Der Bericht aus der Schweiz erlaubt allerdings nur die in 


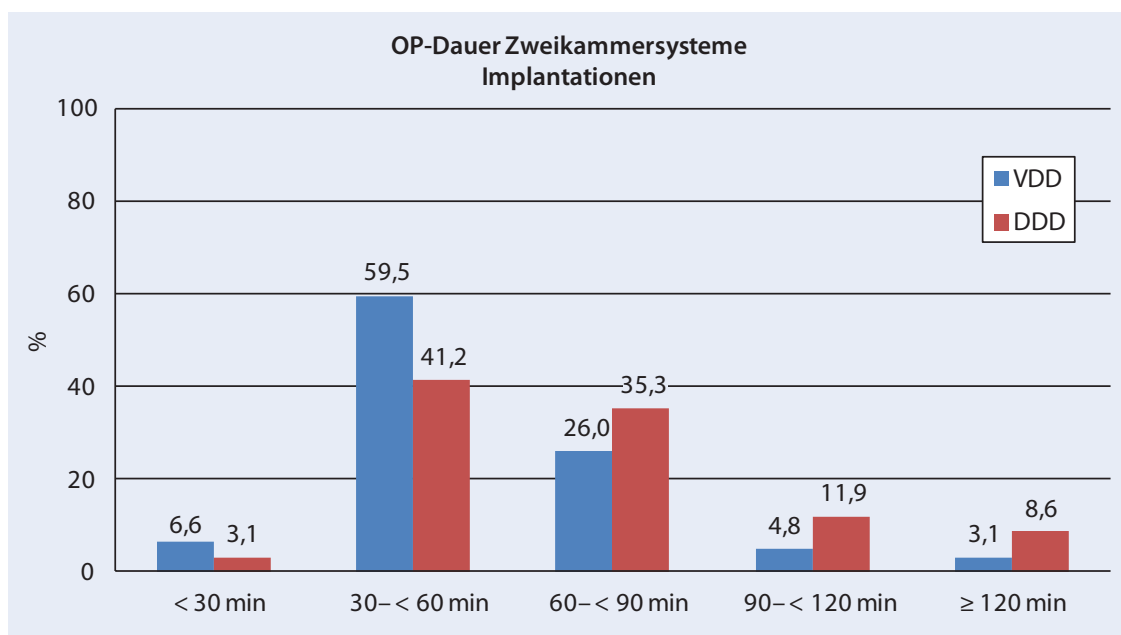

Abb. $7 \Delta$ Prozentuale Verteilung der Operationszeiten bei der Implantation von Zweikammersystemen bezogen auf alle Implantationen von Zweikammersystemen (Beispiel: Bei 41,2\% der implantierten DDD-Systeme lag die Operationsdauer zwischen 30 und $<60 \mathrm{~min}$ )

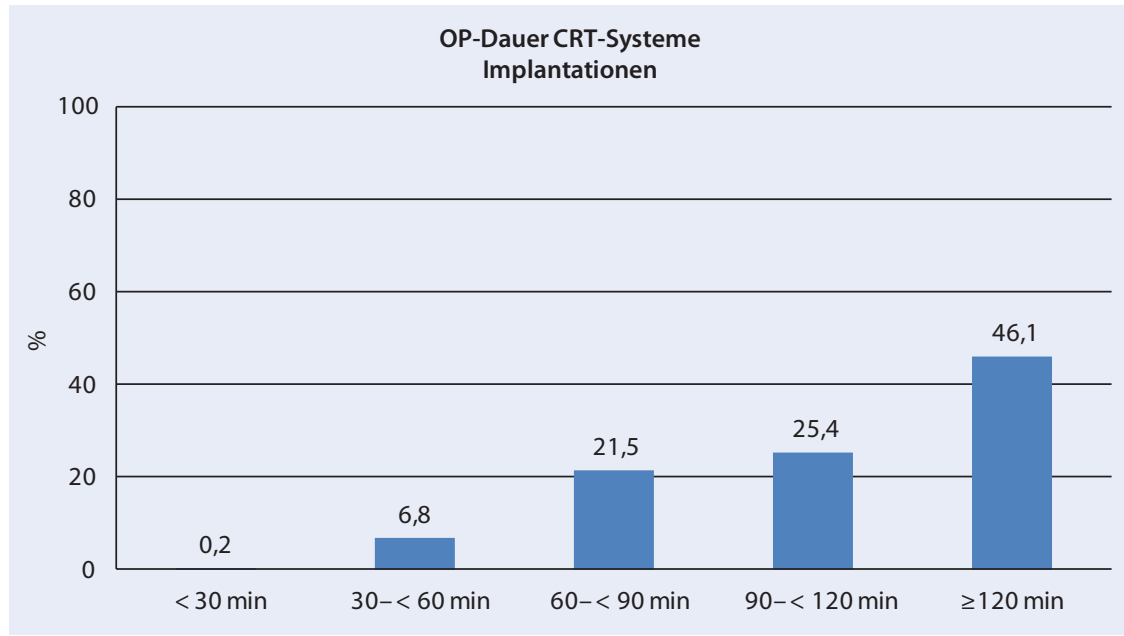

Abb. $8 \Delta$ Prozentuale Verteilung der Operationszeiten bei der Implantation von CRT-Systemen bezogen auf alle Implantationen von CRT-Systemen (Beispiel: Bei 6,8\% der implantierten CRT-Systeme lag die Operationsdauer zwischen 30 und $<60 \mathrm{~min}$ )

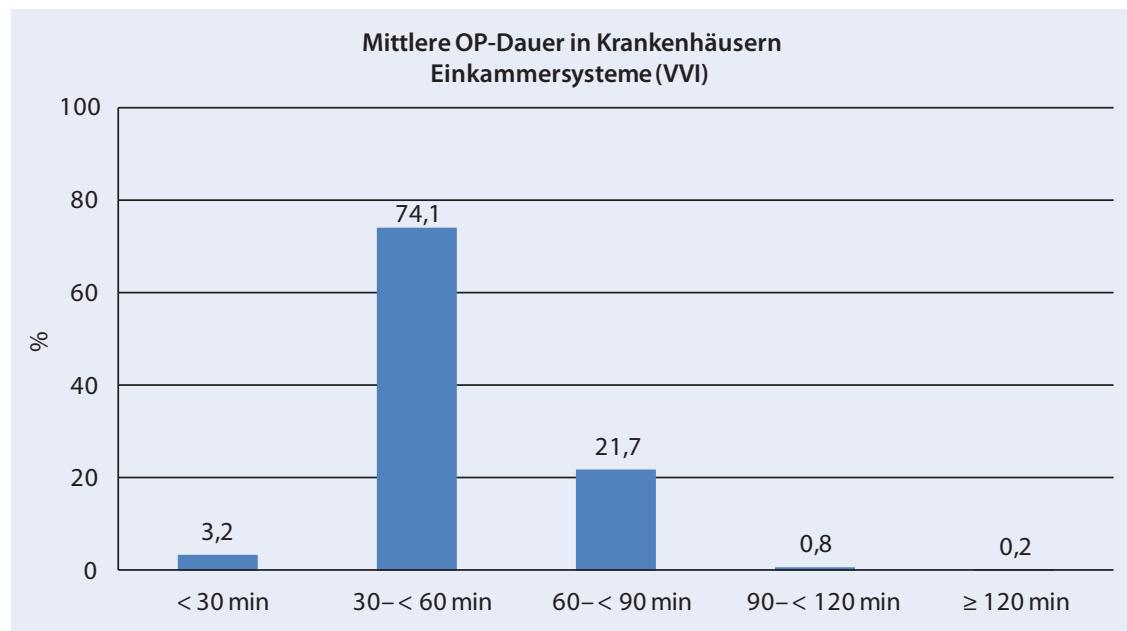

Abb. $9 \Delta$ Prozentuale Verteilung der Operationsdauer bei der Implantation von Einkammersystemen (VVI) bezogen auf die Mittelwerte der Krankenhäuser (Beispiel: Bei 3,2\% aller Krankenhäuser lag der Mittelwert der Operationsdauer einer VVI-Implantation bei $<30 \mathrm{~min}$ )
- Tab. 29 dargestellten Vergleiche, der schwedische Bericht ist in dieser Hinsicht ergiebiger.

Beim Vergleich der Datenbasis zeigt sich zunächst ein ähnliches Bild wie bei den Herzschrittmachern: In Deutschland werden sowohl absolut als auch relativ die meisten ICDs implantiert (• Tab. 29 und - Abb. 17). Es ist weiter davon auszugehen, dass Deutschland hier weltweit führend ist , möglicherweise zusammen mit den USA und Italien, wobei die Datenqualität in diesen beiden Ländern der deutschen nicht wirklich entspricht.

Bei der Suche nach einer Erklärung für diese hohe Implantationsrate wurde zunächst die Altersverteilung betrachtet (- Abb. 18) und tatsächlich ist der Anteil der älteren Patienten, die ein ICD-System erhalten, in Deutschland höher als in Schweden, was aber als Erklärung nicht ausreicht, zumal die Altersstruktur in allen 3 Ländern durchaus vergleichbar ist [11-13].

Der Blick auf die regionale Verteilung zeigt ein Bild, das mit dem bei der Schrittmachertherapie vergleichbar ist, und die Implantationszahlen pro 1 Mio. Einwohner haben in allen Bundesländern bis auf Bremen im Vergleich zum Vorjahr zugenommen (- Abb. 19 und Anhang 2 - Tab. 17).

Die Leitlinienkonformität liegt bundesweit noch nicht vollständig im Bereich der erwarteten Ergebnisse; sie ist am höchsten in Rheinland-Pfalz und in Bayern, Mecklenburg-Vorpommern, Hessen und Sachsen am niedrigsten ( $\bullet$ Abb. 20). Im Vergleich zum Vorjahr haben sich die Zahlen jedoch in allen Bundesländern zumeist im zweistelligen Bereich nach oben entwickelt und damit die im letzten Bericht vom Verfasser prognostizierte Entwicklung genommen.

Wie bei den Herzschrittmacherimplantationen müssen wir auch bei der ICD-Implantationsrate eine schlüssige Erklärung für den deutlichen Unterschied im Vergleich zu unseren europäischen Nachbarn schuldig bleiben. Dies kann 2 Ursachen haben:

1. Die erhobenen Daten sind ungeeignet, um die Unterschiede zu erklären.

2. Die erhobenen Daten bilden die Behandlungswirklichkeit nicht $\mathrm{ab}$. 


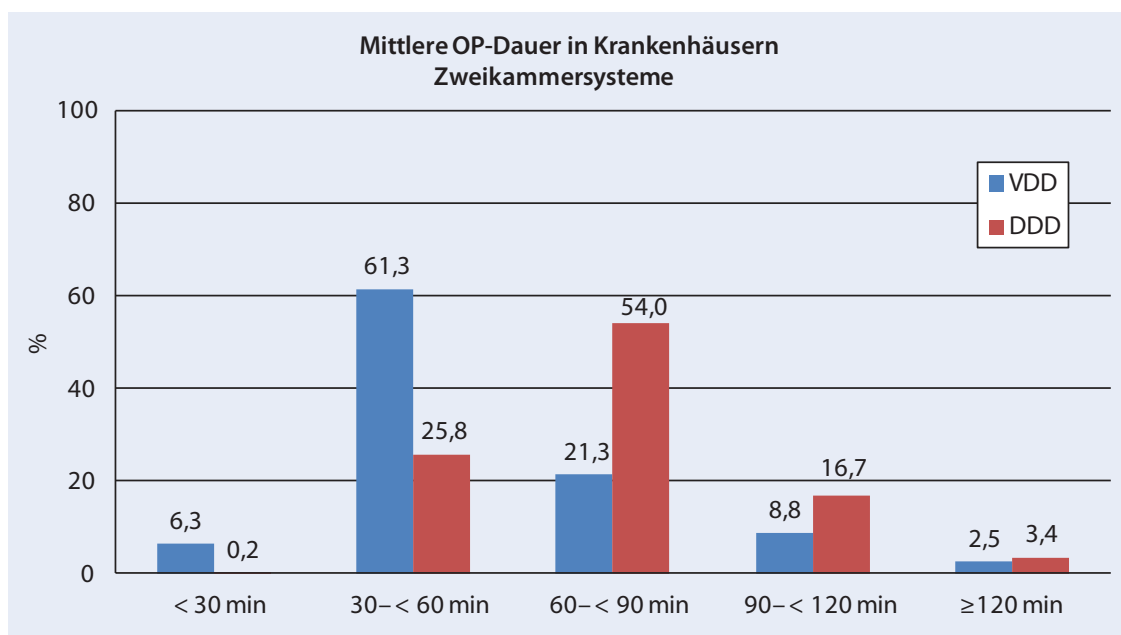

Abb. $10 \Delta$ Prozentuale Verteilung der Operationsdauer bei der Implantation von Zweikammersystemen bezogen auf die Mittelwerte der Krankenhäuser (Beispiel: Bei 0,2\% aller Krankenhäuser lag der Mittelwert der Operationsdauer einer DDD-Implantation bei $<30 \mathrm{~min}$ )

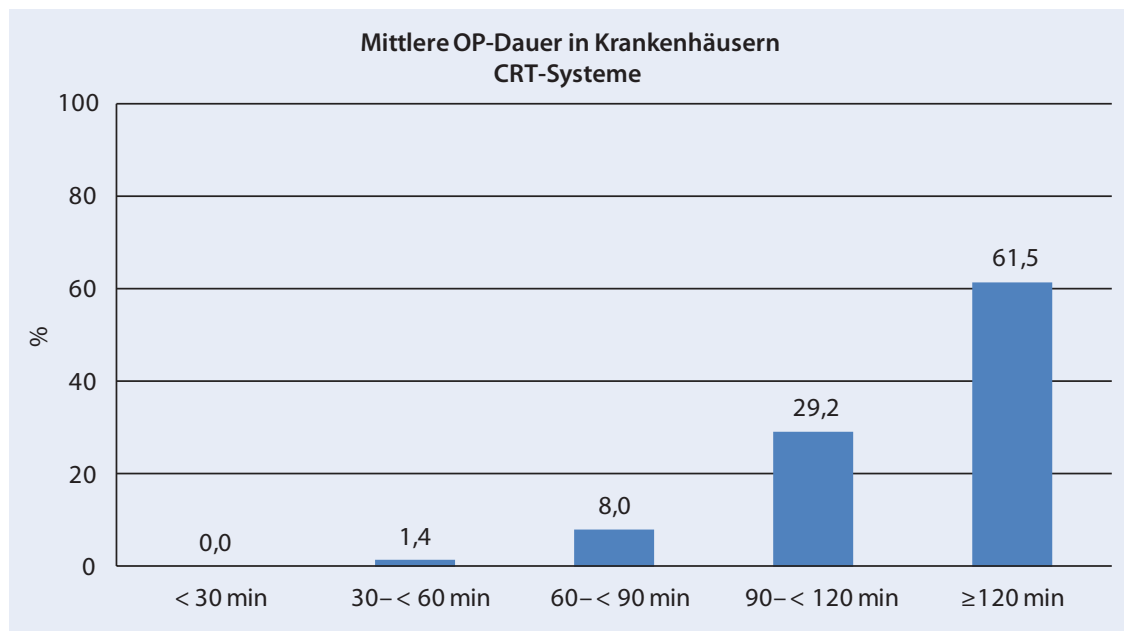

Abb. 11 A Prozentuale Verteilung der Operationsdauer bei der Implantation von CRT-Systemen bezogen auf die Mittelwerte der Krankenhäuser (Beispiel: Bei 0,0\% aller Krankenhäuser lag der Mittelwert der Operationsdauer einer CRT-Implantation bei $<30 \mathrm{~min}$ )

Damit bleibt es Spekulation, welchem der beiden im Folgenden genannten Szenarien man den Vorzug geben möchte, wobei relativ klar ist, welcher Player im Gesundheitswesen sich welche Spekulation zu eigen machen wird:

1. Die Implantationsrate in Deutschland entspricht dem tatsächlichen Bedarf, d. h. die Patienten in der Schweiz und in Schweden sind im Bereich der Therapie mit kardialen Rhythmusimplantaten unterversorgt.

2. Die Implantationsrate in Deutschland entspricht einer Überversorgung der Patienten. men ist die logische Konsequenz. Eine Bewertung dieser Unterschiede ist aufgrund fehlender Daten im schwedischen Register schwierig. Aus den USA sind ähnliche Ergebnisse bekannt, ohne dass sich dort eine überzeugende Erklärung hätte finden lassen [14]. Insgesamt erscheint die ICDSystemauswahl in Deutschland eher dem aktuellen Wissensstand zu entsprechen.

Bei der ICD-Sondenauswahl ist das Vorgehen der Schweden einleuchtender als das in Deutschland: Sie implantieren deutlich weniger Dual-coil-ICD-Sonden und bevorzugen stattdessen die Single-coil-Variante (• Abb. 23).

\section{Operationsdaten}

Auch bei den Operationsdaten sind die Ergebnisse bzw. deren Unterschiede konstant: Die Schweden verwenden wie bei den Schrittmacherimplantationen die V.cephalica deutlich häufiger zum Sondenvorschub als die Deutschen ( $\bullet$ Tab. 30) und operieren schneller (• Abb. 24). Bei den perioperativen Komplikationen schneiden die Deutschen besser ab ( $\bullet$ Abb. 25), wobei die unterschiedlichen Nachbeobachtungszeiträume eine Rolle spielen könnten.

\section{Zusammenfassung und Ausblick}

Sowohl die erwarteten als auch die unerwarteten Ergebnisse des ersten ICDRegisterberichts zeigen eine beachtliche Konstanz: Die Implantationsrate bleibt sehr hoch und steigt weiter an, die Leitlinienkonformität hat erwartungsgemäß deutlich zugenommen. Die Implantationsrate an Dual-coil-Sonden bleibt hoch, zeigt aber eine abnehmende Tendenz, und die Verwendung der Vena cephalica wird immer seltener.

Auf den Zusammenhang zwischen der immer beliebteren Technik der Sondenimplantation mittels Punktion der V. subclavia und deren methodenimmanenten Komplikationen weisen wir bei den Schrittmacheroperationen schon seit Jahren hin, ebenso wie auf den Zusammenhang zwischen Anzahl der Operationen und Komplikationen, wobei diese Ergebnisse offensichtlich nicht zur Kenntnis genommen werden.

Weiter besorgniserregend hoch ist die Rate an Sondenkomplikationen, nicht zu- 


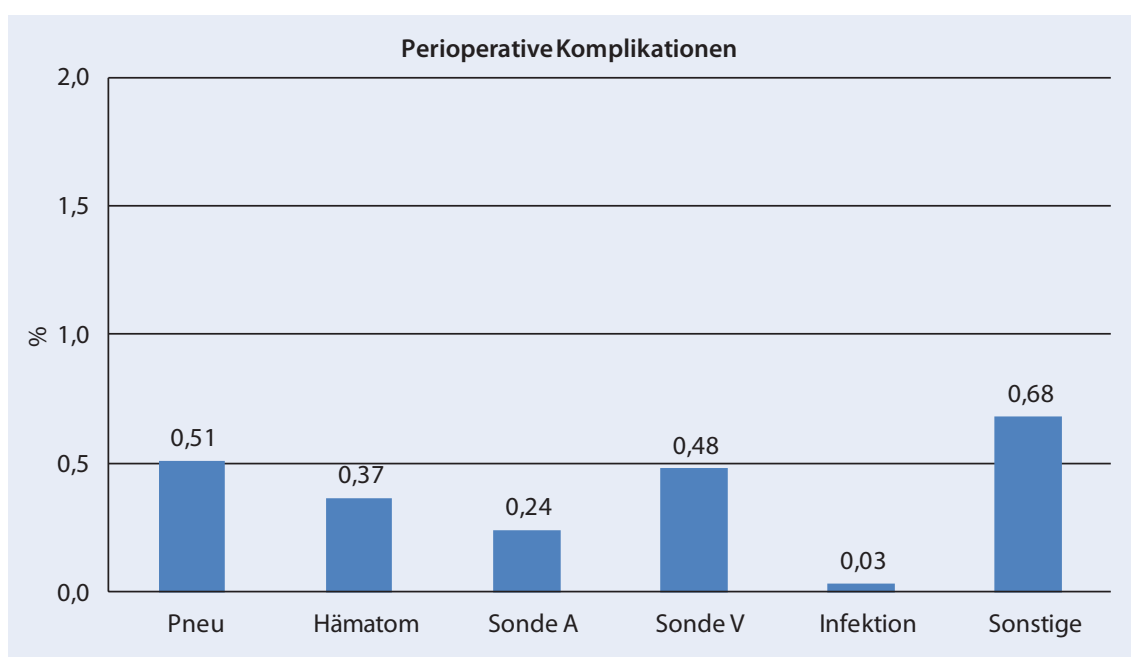

Abb. 12 U Überblick über die perioperativen Komplikationen nach Implantation (Pneu interventionspflichtiger Pneumothorax, Hämatom interventionspflichtiges Taschenhämatom, Sonde A Sondendislokation der Vorhofelektrode, Sonde V Sondendislokation der Ventrikelelektrode, Infektion postoperative Wundinfektion nach Definition der CDC, Sonstige Fälle mit mindestens einer der folgenden perioperativen Komplikationen: kardiopulmonale Reanimation, interventionspflichtiger Perikarderguss, interventionspflichtiger Hämatothorax, Sondendislokation anderer Defibrillationssonden, Sondendysfunktion oder sonstige interventionspflichtige perioperative Komplikationen)

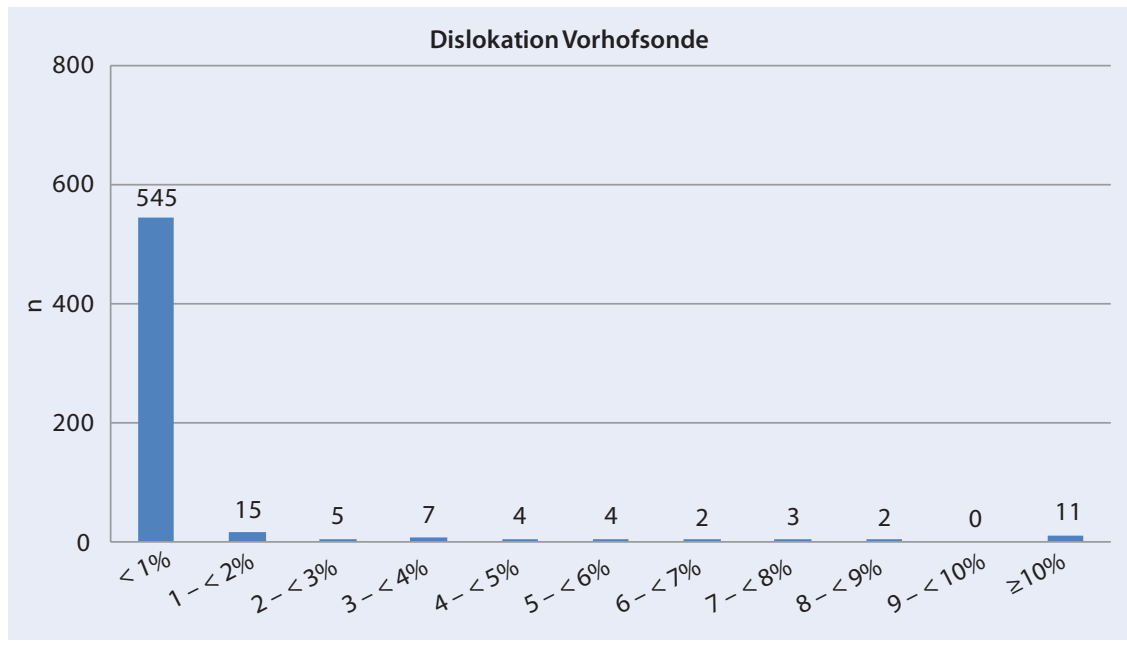

Abb. $13 \Delta$ Verteilung der Häufigkeit von Dislokationen der Vorhofsonde je Krankenhaus bei Implantationen (Beispiel: 545 Krankenhäuser wiesen in unter 1\% ihrer Fälle eine Vorhofsondendislokation auf; Basis der Prozentberechnung sind alle implantierten Systeme mit Vorhofsonde)

letzt bedingt durch konstruktionsbedingte Probleme. Hier gibt es noch einigen Diskussionsbedarf auch hinsichtlich der Produktüberwachung, wobei auch hier ein Interesse an einer diesbezüglichen Diskussion außerhalb von Expertenrunden nicht wirklich erkennbar ist.

Im letzten Jahr haben wir 3 Parameter vorgestellt, die über die Zeit betrachtet werden sollen. Die Ergebnisse sind in - Tab. 31 aufgeführt und wurden bis auf den Punkt 3 bereits weiter oben kommentiert. Der Rückgang der Zahlen bei unver- ständlich hohen Implantationsraten von Dual-coil-Sonden ist dabei ebenso erfreulich wie die ebenfalls abnehmende Rate an Häusern, in denen eine Dislokation der rechtsventrikulären Sonde häufiger als in $5 \%$ der Fälle auftrat.

Die Leser des 1 . Teils werden die folgenden Worte schon kennen, für diejenigen, die nur Teil 2 gelesen haben, sei an dieser Stelle unser herzlicher Dank an alle, die zu diesem Bericht beigetragen haben, insbesondere an die Mitarbeiterinnen und Mitarbeitern beim AQUA-Ins- titut nochmals betont und der Hoffnung Ausdruck verliehen, dass auch die Informationen des Teils 2 des Registerberichts 2011 dem Anwender in der täglichen Praxis hilfreich sein können.

\section{Korrespondenzadresse}

\section{OTA Prof. Dr. A. Markewitz}

Abt. XVII - Herz- und Gefäßchirurgie

Bundeswehrzentralkrankenhaus

Rübenacher Str. 170, 56072 Koblenz

AndreasMarkewitz@bundeswehr.org

\section{Literatur}

1. http://www.pacemaker-register.de

2. http://www.pacemaker.ch/download/statistiken/ ch_stat_2011.pdf. Zugegriffen: 18. Mär. 2013

3. https://www.pacemakerregistret.se/icdpmr/docbankView.do?id=0__IXU--IZEOKKnOwSrr8. Zugegriffen: 18. Mär. 2013

4. Rinaldi CA, Simon RDB, Geelen P et al (2003) A randomized prospective study of single coil versus dual coil defibrillation in patients with ventricular arrhythmias undergoing implantable cardioverter defibrillator therapy. PACE 26:1684-1690

5. Theuns DAMJ, Szili-Torok T, Jordaens LJ (2005) Defibrillation efficacy testing: long-term follow-up and mortality. Europace 7:509-515

6. Vischer AS, Sticherling C, Kühne MS, Osswald S, Schaer BA (2013) Role of defibrillation threshold testing in the contemporary defibrillator patient population. J Cardiovasc Electrophysiol 24:437441

7. van Rees JB, Borleffs CJW, de Bie MK et al (2011) Inappropriate implantable cardioverter-defibrillator shocks Incidence, predictors, and impact on mortality. J Am Coll Cardiol 57:556-562

8. Germano JJ, Reynolds M, Essebag V, Josephson ME (2006) Frequency and causes of implantable cardioverter-defibrillator therapies: is device therapy proarrhythmic? Am J Cardiol 97:1255-1261

9. Birnie DH, Parkash R, Exner ED et al (2012) Clinical predictors of fidelis lead failure: a report from the canadian heart rhythm society device committee. Circulation 125:1217-1225

10. Hauser RG (2012) Here we go again - another failure of postmarketing device surveillance. $\mathrm{N}$ Engl J Med 366:873-875

11. http://www.bfs.admin.ch/bfs/portal/de/index/ themen/01/02/blank/key/alter/gesamt.html. Zugegriffen: 11. Apr. 2013

12. http://www.scb.se/Pages/StatisticStart 351774.aspx. Zugegriffen: 11. Apr. 2013

13. https://www-genesis.destatis.de/genesis/online/ data;jsessionid=D08971CE69172142A40D9D889 78790F9.tomcat_GO_2_2?operation=abruftabe IleBearbeiten\&levelindex $=2 \&$ levelid $=136568013$ 6329\&auswahloperation=abruftabelleAuspraeg ungAuswaehlen\&auswahlverzeichnis=ordnung sstruktur\&auswahlziel=werteabruf\&selectionna $\mathrm{me}=12411-0005 \&$ auswahltext $=\&$ werteabruf $=$ star ten. Zugegriffen: 11. Apr. 2013

14. Matlock DD, Peterson PN, Wang Y et al (2012) Variation in use of dual-chamber implantable cardioverter-defibrillators: results from the national cardiovascular data registry. Arch Intern Med 172:634-641 
Tab. 17 Perioperative Komplikationen bei Implantationen in den Jahren 2010 und 2011 im Vergleich

\begin{tabular}{|c|c|c|c|c|}
\hline & \multicolumn{2}{|l|}{2010} & \multicolumn{2}{|l|}{2011} \\
\hline & $\mathrm{n}$ & Prozent & $\mathrm{n}$ & Prozent \\
\hline Mindestens eine perioperative Komplikation & 620 & 2,4 & 625 & 2,2 \\
\hline Kardiopulmonale Reanimation & 37 & 0,1 & 46 & 0,2 \\
\hline Interventionspflichtiger Pneumothorax & 117 & 0,5 & 144 & 0,5 \\
\hline Interventionspflichtiger Perikarderguss & 31 & 0,1 & 38 & 0,1 \\
\hline Interventionspflichtiges Taschenhämatom & 109 & 0,4 & 104 & 0,4 \\
\hline Interventionspflichtiger Hämatothorax & 16 & 0,1 & 21 & 0,1 \\
\hline Postoperative Wundinfektion (nach Definition der CDC) & 15 & 0,1 & 9 & $<0,1$ \\
\hline Sonstige interventionspflichtige Komplikationen & 52 & 0,2 & 45 & 0,2 \\
\hline \multicolumn{5}{|l|}{ Sondendislokation } \\
\hline Bezogen auf alle Patienten & 220 & 0,9 & 199 & 0,7 \\
\hline - Nur Vorhofsonde & 70 & 0,3 & 63 & 0,2 \\
\hline - Nur Ventrikelsonde & 142 & 0,6 & 131 & 0,5 \\
\hline - Vorhof- und Ventrikelsonde & 8 & $<0,1$ & 5 & $<0,1$ \\
\hline - Andere Defibrillationssonde & 0 & 0,0 & 0 & 0,0 \\
\hline \multicolumn{5}{|l|}{ Bezogen auf Patienten mit Vorhof- bzw. Ventrikelsonde } \\
\hline -Vorhofsonde & $78 / 13.826$ & 0,6 & $68 / 15.793$ & 0,4 \\
\hline -Ventrikelsonde & $150 / 25.582$ & 0,6 & $136 / 28.452$ & 0,5 \\
\hline \multicolumn{5}{|l|}{ Sondendysfunktion } \\
\hline Bezogen auf alle Patienten & 70 & 0,3 & 55 & 0,2 \\
\hline - Nur Vorhofsonde & 11 & $<0,1$ & 3 & $<0,1$ \\
\hline - Nur Ventrikelsonde & 55 & 0,2 & 51 & 0,2 \\
\hline - Vorhof- und Ventrikelsonde & 4 & $<0,1$ & 1 & $<0,1$ \\
\hline - Andere Defibrillationssonde & 1 & $<0,1$ & 0 & 0,0 \\
\hline \multicolumn{5}{|l|}{ Bezogen auf Patienten mit Vorhof- bzw. Ventrikelsonde } \\
\hline -Vorhofsonde & $15 / 13.826$ & 0,1 & $4 / 15.793$ & $<0,1$ \\
\hline - Ventrikelsonde & $59 / 25.582$ & 0,2 & $52 / 28.452$ & 0,2 \\
\hline
\end{tabular}

Tab. 18 Perioperative Komplikationen in Abhängigkeit vom venösen Zugangsweg für den Sondenvorschub

\section{Komplikation 2011}

Kardiopulmonale Reanimation

Interventionspflichtiger Pneumothorax

Interventionspflichtiger Perikarderguss

Interventionspflichtiges Taschenhämatom

Interventionspflichtiger Hämatothorax

Sondendislokation

Sondendysfunktion

Postoperative Wundinfektion nach Definition der CDC

Sonstige interventionspflichtige perioperative Komplikation

Mindestens eine perioperative Komplikation

Fisher's Test: ${ }^{*} p<0,05 ;{ }^{* *} p<0,01 ;{ }^{* * *} p<0,001$

\section{Zugang nur über Vena Zugang nur über Vena Signifikanz} cephalica (\%)

\begin{tabular}{lll}
0,20 & 0,14 & $* * *$ \\
0,16 & 0,60 & $*$ \\
\hline 0,09 & 0,15 & \\
0,26 & 0,41 & $*$ \\
0,04 & 0,08 & \\
0,46 & 0,73 & \\
0,17 & 0,20 & $* * *$ \\
0,01 & 0,04 & $*$ \\
\hline 0,16 & 0,17 & \\
\hline 1,45 & 2,40 & \\
\hline
\end{tabular}




\section{Deutsches Herzschrittmacher- und Defibrillator-Register}

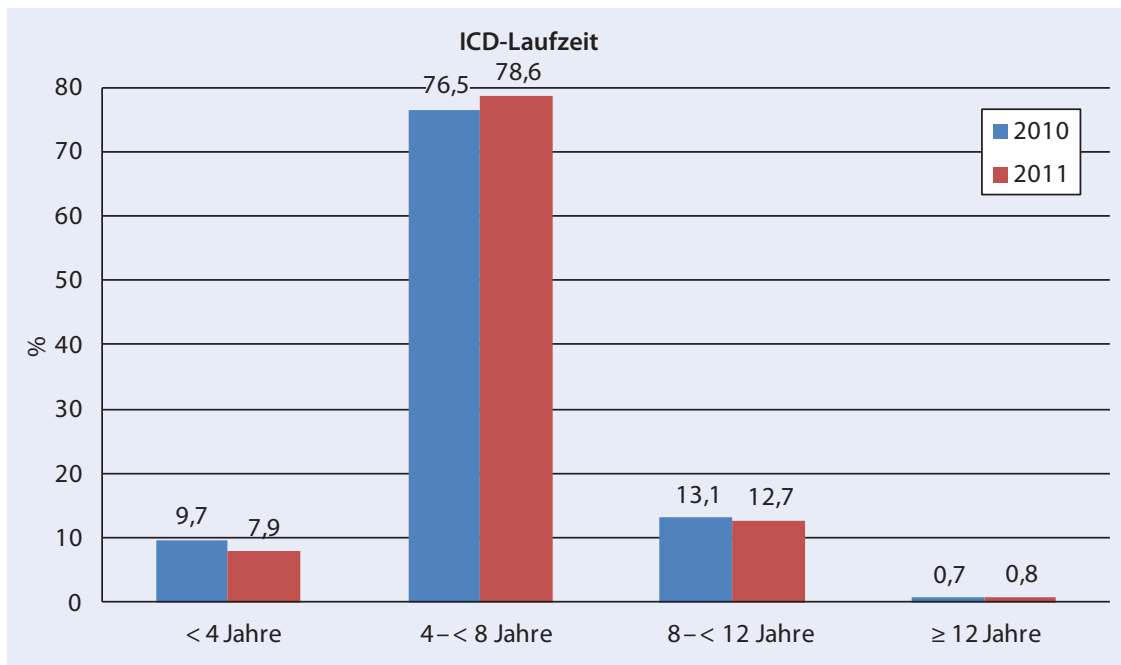

Abb. 14 A Verteilung der Aggregatlaufzeiten (Beispiel: 2011 lag bei 78,6\% der Austauscheingriffe mit Laufzeitangabe die Laufzeit zwischen 4 und $<8$ Jahren)

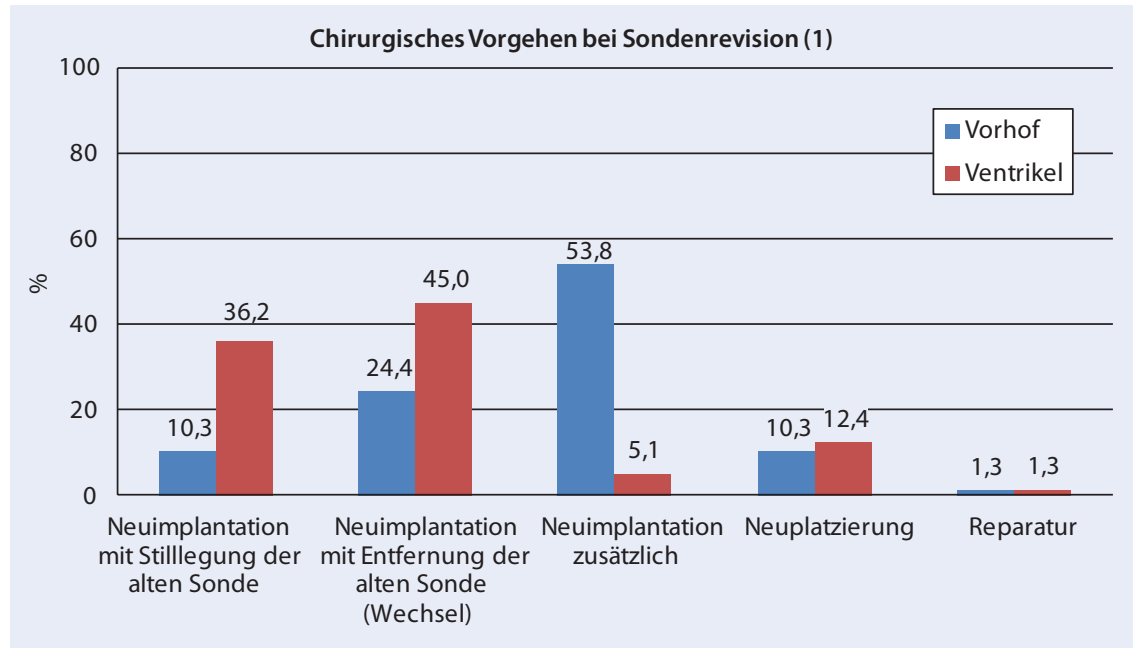

Abb. $15 \Delta$ Chirurgisches Vorgehen bei der Sondenrevision. Bezug: alle postoperativ funktionell aktiven Sonden (1. Sonde), an denen ein Eingriff vorgenommen wurde

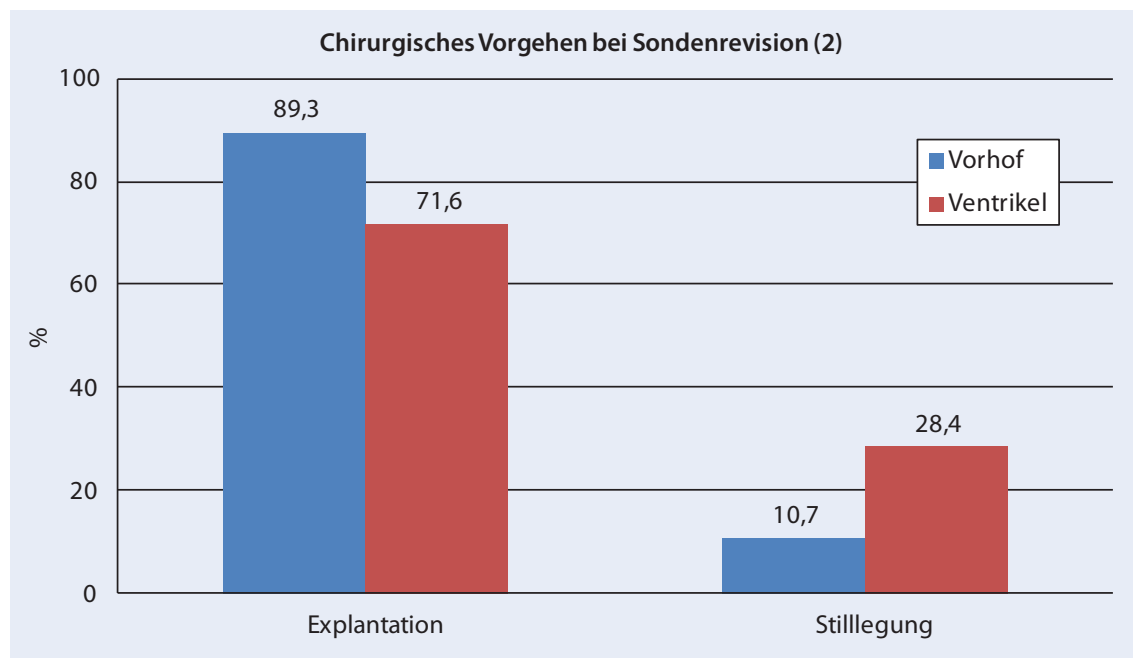

Abb. $16 \Delta$ Chirurgisches Vorgehen bei funktionslosen Sonden. Bezug: alle postoperativ funktionell nicht aktiven Sonden (1. Sonde), bei denen die Art des Vorgehens dokumentiert wurde 
Tab. 19 Perioperative Komplikationen in Abhängigkeit vom venösen Zugangsweg für den Sondenvorschub nach Fallzahlklassen

Perioperative Komplikationen 2011

Zugang nur über Vena cephalica

Fallzahlklassen (\%)

Kardiopulmonale Reanimation

Interventionspflichtiger Pneumothorax

Interventionspflichtiger Perikarderguss

Interventionspflichtiges Taschenhämatom

Interventionspflichtiger Hämatothorax

Sondendislokation

Sondendysfunktion

Postoperative Wundinfektion nach Definition der CDC

Sonstige interventionspflichtige perioperative Komplikation

Mindestens eine perioperative Komplikation

\begin{tabular}{llllll}
$\mathbf{5} 50$ & $\mathbf{5 1 - 1 0 0}$ & $>100$ & $\mathbf{5 0}$ & $\mathbf{5 1 - 1 0 0}$ & $>100$ \\
\hline 0,27 & 0,24 & 0,09 & 0,21 & 0,07 & 0,14 \\
\hline 0,35 & 0,08 & 0,05 & 0,57 & 0,59 & 0,62 \\
\hline 0,18 & 0,04 & 0,05 & 0,13 & 0,16 & 0,16 \\
\hline 0,58 & 0,16 & 0,05 & 0,49 & 0,33 & 0,43 \\
\hline 0,04 & 0,04 & 0,05 & 0,11 & 0,05 & 0,09 \\
\hline 0,71 & 0,32 & 0,37 & 0,82 & 0,75 & 0,65 \\
\hline 0,22 & 0,08 & 0,23 & 0,10 & 0,24 & 0,23 \\
\hline 0,04 & 0,00 & 0,00 & 0,06 & 0,02 & 0,06 \\
\hline 0,18 & 0,16 & 0,14 & 0,19 & 0,23 & 0,10 \\
\hline 2,40 & 1,12 & 0,83 & 2,53 & 2,33 & 2,37
\end{tabular}

Tab. 20 Laufzeit der ICD-Aggregate in Jahren bezogen auf das ICD-System

\begin{tabular}{|lllllllll}
\hline & Anzahl $(\boldsymbol{n})$ & MW & \multicolumn{3}{c}{ SD } & \multicolumn{3}{c}{ Median } \\
\hline & $\mathbf{2 0 1 0}$ & $\mathbf{2 0 1 1}$ & $\mathbf{2 0 1 0}$ & $\mathbf{2 0 1 1}$ & $\mathbf{2 0 1 0}$ & $\mathbf{2 0 1 1}$ & $\mathbf{2 0 1 0}$ & $\mathbf{2 0 1 1}$ \\
\hline VVI & 2456 & 2758 & 6,3 & 6,4 & 2,0 & 1,9 & 6 & 6 \\
\hline VDD & 31 & 47 & 5,4 & 5,5 & 1,8 & 1,8 & 5 & 5 \\
\hline DDD & 1471 & 1621 & 5,5 & 5,7 & 1,7 & 1,6 & 5 & 6 \\
\hline CRT & 1825 & 2158 & 4,5 & 4,6 & 1,3 & 1,3 & 5 & 5 \\
\hline
\end{tabular}

Datensatz 09/5 Defibrillatoren-Aggregatwechsel, nur gültige Angaben zur Lebensdauer und zum implantierten ICD-System wurden ausgewertet

Tab. 21 Häufigkeit der Therapieabgabe der ausgetauschten ICD-Aggregate

\begin{tabular}{lllll}
\hline Therapien abgegeben? & $\mathbf{2 0 1 0}$ & & $\mathbf{2 0 1 1}$ & \\
\hline & $\boldsymbol{n}$ & Prozent & $\boldsymbol{n}$ & Prozent \\
\hline Nein & 3361 & 56,0 & 4052 & 59,4 \\
\hline Adäquat & 2347 & 39,1 & 2452 & 36,0 \\
\hline Inadäquat & 145 & 2,4 & 163 & 2,4 \\
\hline Beides & 149 & 2,5 & 151 & 2,2 \\
\hline
\end{tabular}

Tab. 22 Durchführung des intraoperativen Defibrillationstests (Aggregatwechsel)

\begin{tabular}{|lllll}
\hline $\begin{array}{l}\text { Intraoperativer Defibrillationstest } \\
\text { bei Aggregatwechsel durchgeführt? }\end{array}$ & $\mathbf{2 0 1 0}$ & & $\mathbf{2 0 1 1}$ \\
\cline { 2 - 5 } & $\boldsymbol{n}$ & Prozent & $\boldsymbol{n}$ & Prozent \\
\hline Ja & 2916 & 48,6 & 2607 & 38,2 \\
\hline Sicherheitsabstand: nein & 90 & 1,5 & 94 & 1,4 \\
\hline Sicherheitsabstand: ja & 2826 & 47,1 & 2513 & 36,9 \\
\hline Nein, wegen intrakardialen Thromben & 239 & 4,0 & 290 & 4,3 \\
\hline $\begin{array}{l}\text { Nein, wegen hämodynamischer In- } \\
\begin{array}{l}\text { stabilität (katecholaminpflichtig oder } \\
\text { Lungenödem) }\end{array}\end{array}$ & 133 & 2,2 & 178 & 2,6 \\
\hline Nein, aus sonstigen Gründen & 2714 & 45,2 & 3743 & 54,9 \\
\hline
\end{tabular}

Tab. 23 Ort des letzten Eingriffs, welcher der Revisionsoperation vorausging

\begin{tabular}{|lllll}
\hline Ort des letzten Eingriffs & $\mathbf{2 0 1 0}$ & & $\mathbf{2 0 1 1}$ & \\
\hline & $\boldsymbol{n}$ & Prozent & $\boldsymbol{n}$ & Prozent \\
\hline Eigene Institution & 5068 & 72,3 & 5755 & 71,4 \\
\hline Andere Institution & 1946 & 27,7 & 2301 & 28,6 \\
\hline Summe & 7014 & 100,0 & 8056 & 100,0 \\
\hline
\end{tabular}




\section{Deutsches Herzschrittmacher- und Defibrillator-Register}

\begin{tabular}{lllll}
$\begin{array}{l}\text { Tab. 24 Indikation zur Revisionsoperation (Mehrfachnennung möglich) } \\
\text { Indikation zur Revision }\end{array}$ & $\mathbf{2 0 1 0}$ & & $\mathbf{2 0 1 1}$ & \\
\hline & $\boldsymbol{n}$ & Prozent & $\boldsymbol{n}$ & Prozent \\
\hline Infektion & 985 & 14,0 & 1088 & 13,5 \\
\hline Taschenproblem & 662 & 9,4 & 768 & 9,5 \\
\hline Aggregatproblem & 2082 & 29,7 & 2288 & 28,4 \\
\hline Systemumwandlung & 1738 & 24,8 & 2131 & 26,5 \\
\hline Sondenproblem & 3792 & 54,1 & 4250 & 52,8 \\
\hline Ineffektive Defibrillation & 113 & 1,6 & 110 & 1,4 \\
\hline Sonstiges & 819 & 11,7 & 776 & 9,6
\end{tabular}

Tab. 25 Indikation zur Revisionsoperation bei Aggregatproblemen bzw. Systemumwandlungen (Mehrfachnennungen möglich)

\begin{tabular}{|c|c|c|c|}
\hline & 2010 & 2011 & \\
\hline & $\%$ aller Revisionen & $n$ & $\%$ aller Revisionen \\
\hline \multicolumn{4}{|l|}{ Indikationen zum Aggregatwechsel } \\
\hline Aggregatproblem & 29,7 & 2288 & 28,4 \\
\hline Batterieerschöpfung & 16,3 & 1334 & 16,6 \\
\hline Fehlfunktion/Rückruf & 1,5 & 101 & 1,3 \\
\hline $\begin{array}{l}\text { Vorzeitiger Aggregataustausch an- } \\
\text { lässlich einer Revisionsoperation }\end{array}$ & 4,9 & 375 & 4,7 \\
\hline Sonstige Indikation & 6,9 & 478 & 5,9 \\
\hline \multicolumn{4}{|l|}{ Systemumwandlungen } \\
\hline Systemumwandlung (Modul 09/6) & 24,8 & 2131 & 26,5 \\
\hline Zwischen ICD-Systemen & 24,2 & 2092 & 26,0 \\
\hline Vom Defibrillator zum Schrittmacher & 0,6 & 39 & 0,5 \\
\hline Systemumwandlung (Modul 09/4) & & & $\%$ aller Implantationen \\
\hline Vom Schrittmacher zum Defibrillator & 5,0 & 1504 & 5,3 \\
\hline
\end{tabular}

Tab. 26 Indikation zur Revisionsoperation bei Sondenproblemen

\begin{tabular}{|c|c|c|c|c|c|c|c|}
\hline Sondenprobleme & 2010 & $2011(n=8$ & & & & & \\
\hline & Gesamt (\%) & $\begin{array}{l}\text { Vorhof } \\
\text { Sonde (\%) }\end{array}$ & $\begin{array}{l}\text { Ventrikel } 1 . \\
\text { Sonde (\%) }\end{array}$ & $\begin{array}{l}\text { Ventrikel } 2 . \\
\text { Sonde (\%) }\end{array}$ & $\begin{array}{l}\text { Ventrikel } 3 . \\
\text { Sonde (\%) }\end{array}$ & $\begin{array}{l}\text { Andere } \\
\text { Sonde (\%) }\end{array}$ & $\begin{array}{l}\text { Mindestens eine Son- } \\
\text { de durch genanntes } \\
\text { Problem betroffen }\end{array}$ \\
\hline Dislokation & 14,0 & 5,1 & 6,1 & 4,0 & 0,2 & 0,1 & 13,9 \\
\hline Sondenbruch/lsolationsdefekt & 18,8 & 2,7 & 15,2 & 2,2 & 0,2 & 0,1 & 17,9 \\
\hline Zwerchfellzucken & 1,6 & 0,1 & 0,3 & 1,3 & 0,1 & - & 1,6 \\
\hline Oversensing & 5,9 & 0,5 & 4,8 & 0,7 & 0,1 & - & 5,2 \\
\hline Undersensing & 4,2 & 0,5 & 3,1 & 0,8 & 0,1 & - & 3,8 \\
\hline $\begin{array}{l}\text { Stimulationsverlust/Reizschwel- } \\
\text { lenanstieg }\end{array}$ & 12,7 & 1,9 & 8,4 & 3,2 & 0,2 & - & 12,1 \\
\hline Perforation & 1,0 & 0,2 & 1,0 & 0,1 & 0,0 & $<0,1$ & 1,2 \\
\hline Infektion & 7,6 & 5,2 & 7,7 & 3,3 & 0,3 & 0,2 & 7,9 \\
\hline Sonstiges & 12,1 & 3,5 & 7,2 & 3,6 & 0,3 & 0,3 & 11,2 \\
\hline $\begin{array}{l}\text { Anteil Patienten mit einem der } \\
\text { aufgeführten Sondenprobleme }\end{array}$ & 72,3 & 19,6 & 53,7 & 19,1 & 1,4 & 0,8 & $70,2^{\mathrm{a}}$ \\
\hline
\end{tabular}




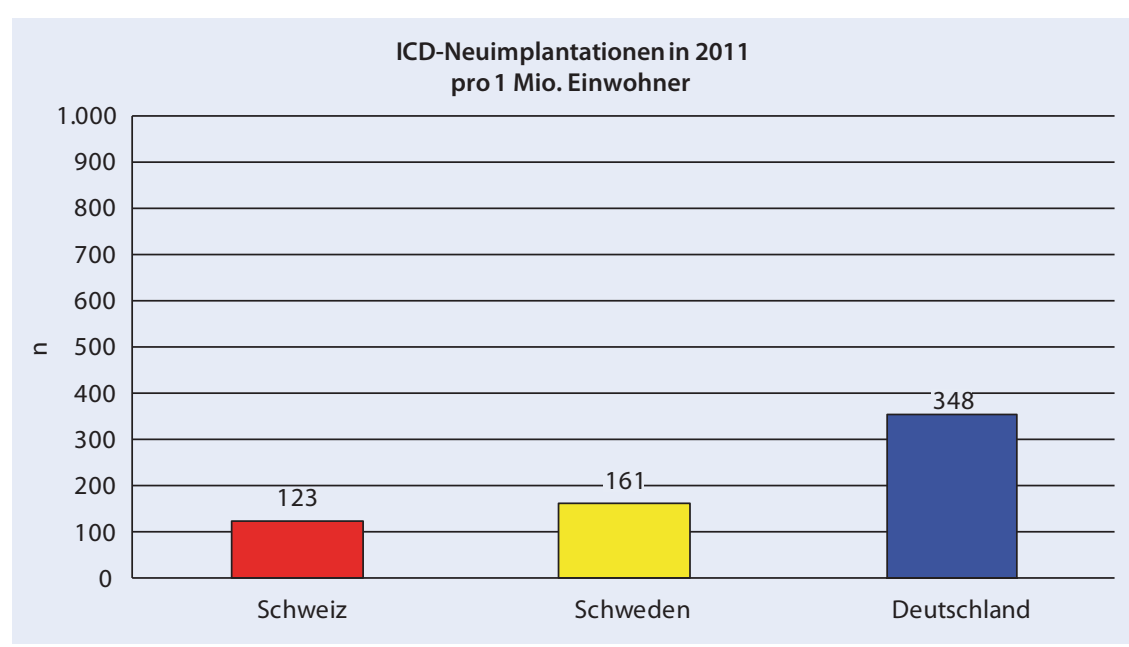

Abb. 17 A Implantationen pro 1 Mio. Einwohner im internationalen Vergleich

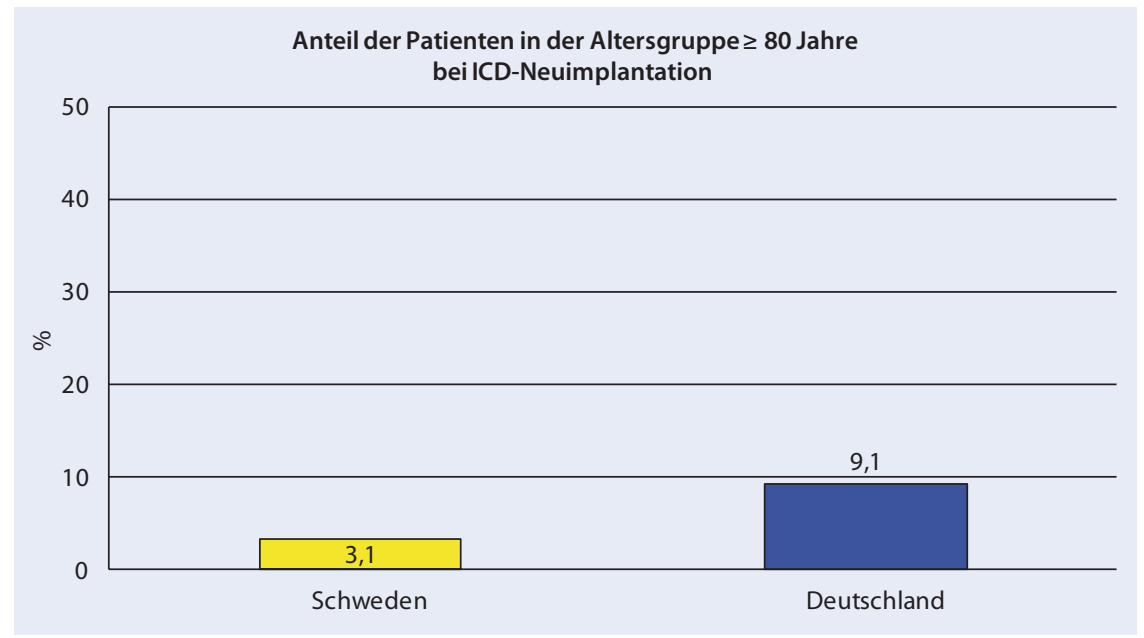

Abb. $18 \Delta$ Anteil älterer Patienten $\geq 80$ Jahre an allen Patienten, bei denen ein ICD implantiert wurde, im Vergleich 


\section{Deutsches Herzschrittmacher- und Defibrillator-Register}

Tab. 27 Komplikationen bei Aggregatwechsel und Revisionen/Systemwechsel/Explantationen 2011 (Vorjahresdaten in Klammern; Basis der

Prozentberechnung sind jeweils alle Aggregatwechsel bzw. Revisionen/Systemwechsel/Explantationen)

\begin{tabular}{lllll} 
& Aggregatwechsel & & \multicolumn{2}{l}{ Revisionen/Systemwechsel/Explantationen } \\
\hline & $n$ & Prozent & $n$ & Prozent \\
\hline Mindestens eine perioperative Komplikation & 50 & $0,73(0,82)$ & 218 & $2,71(2,87)$ \\
\hline Kardiopulmonale Reanimation & 3 & $0,04(0,05)$ & 20 & $0,25(0,24)$ \\
\hline Interventionspflichtiger Pneumothorax & - & - & 54 & $0,67(0,64)$ \\
\hline Interventionspflichtiger Perikarderguss & - & - & 8 & $0,10(0,17)$ \\
\hline Interventionspflichtiges Taschenhämatom & 24 & $0,35(0,50)$ & 40 & $0,50(0,48)$ \\
\hline Interventionspflichtiger Hämatothorax & - & - & 13 & $0,16(0,09)$ \\
\hline Sondendislokation & - & - & 42 & $0,52(0,48)$ \\
\hline -Vorhof & - & - & 13 & $0,16(0,14)$ \\
\hline- Ventrikel & - & - & 26 & $0,32(0,29)$ \\
\hline - Beide & - & - & 2 & $0,02(0,06)$ \\
\hline Sondendysfunktion & - & - & 25 & $0,31(0,47)$ \\
\hline -Vorhof & - & - & 4 & $0,05(0,09)$ \\
\hline -Ventrikel & - & - & 21 & $0,26(0,38)$ \\
\hline- Beide & - & - & 0 & $0,00(0,00)$ \\
\hline Postoperative Wundinfektion nach Definition der CDC & 4 & $0,06(0,10)$ & 8 & $0,10(0,20)$ \\
\hline Sonstige interventionspflichtige Komplikation & 20 & $0,29(0,23)$ & 27 & $0,34(0,41)$ \\
\hline
\end{tabular}

Tab. 28 Todesfälle im Zusammenhang mit Implantationen, Aggregatwechseln und Revisionen/Systemwechseln/Explantationen in 2011 im Vergleich zu den Vorjahresdaten

\begin{tabular}{|lllll|}
\hline Tod bei oder nach... & $\mathbf{2 0 1 0}$ & & $\mathbf{2 0 1 1}$ & \\
\hline & $\boldsymbol{n}$ & Prozent & $\boldsymbol{n}$ & Prozent \\
\hline Neuimplantation & 155 & 0,6 & 176 & 0,6 \\
\hline Aggregatwechsel & 14 & 0,2 & 15 & 0,2 \\
\hline Revision/Systemwechsel/Explantation & 113 & 1,6 & 103 & 1,3 \\
\hline
\end{tabular}

\section{Tab. 29 Datenbasis im internationalen Vergleich}

\begin{tabular}{|llll}
\hline & Schweiz & Schweden & Deutschland \\
\hline Meldende Institutionen & 37 & 31 & 664 \\
\hline Implantierende Institutionen & 37 & 31 & 648 \\
\hline Erstimplantationen & 977 & 1525 & 28.452 \\
\hline - Im Mittel je Institution & 26 & 49 & 44 \\
\hline - Pro 1 Mio. Einwohner & 123 & 161 & 348 \\
\hline Folgeeingriffe & 457 & 423 & 14.874 \\
\hline Verhältnis Erstimplantation/Folgeeingriffe & 2,14 & 3,61 & 1,91 \\
\hline Eingriffe insgesamt (Erstimplantationen + Folgeeingriffe) & 1434 & 1948 & 43.326 \\
\hline
\end{tabular}

Tab. 31 Verhalten der Krankenhäuser bei Implantationen bezüglich des bevorzugten venö-

sen Zugangs, der Auswahl der Defibrillationselektroden sowie der Häufigkeit von Ventrikel-

sondendislokationen in den einzelnen Krankenhäusern im Vergleich der Jahre 2010 und 2011

\begin{tabular}{|c|c|c|c|c|c|}
\hline \multicolumn{2}{|c|}{ Krankenhäuser } & \multicolumn{2}{|l|}{2010} & \multicolumn{2}{|c|}{2011} \\
\hline & & 596 & & 648 & \\
\hline & & $n$ & Prozent & $n$ & Prozent \\
\hline 1 & $\begin{array}{l}\text { Verwendung der Vena } \\
\text { cephalica in }<10 \%\end{array}$ & 244 & 40,9 & 258 & 39,8 \\
\hline 2 & $\begin{array}{l}\text { Verwendung einer Dual-coil- } \\
\text { Sonde in } \geq 90 \%\end{array}$ & 242 & 40,6 & 222 & 34,3 \\
\hline 3 & $\begin{array}{l}\text { Dislokation der Ventrikelson- } \\
\text { de in } \geq 5 \%\end{array}$ & 14 & 2,3 & 11 & 1,7 \\
\hline
\end{tabular}

Tab. 30 Prozentuale Verteilung venöser Zugänge bei Neuimplantationen im Vergleich

\begin{tabular}{|lll}
\hline & Schweden (\%) & $\begin{array}{l}\text { Deutschland } \\
\text { (\%) }\end{array}$ \\
\hline $\begin{array}{l}\text { Vena } \\
\text { cephalica }\end{array}$ & 51,3 & 33,9 \\
\hline $\begin{array}{l}\text { Vena } \\
\text { subclavia }\end{array}$ & 48,2 & 74,5 \\
\hline Andere & 0,5 & 2,3 \\
\hline
\end{tabular}




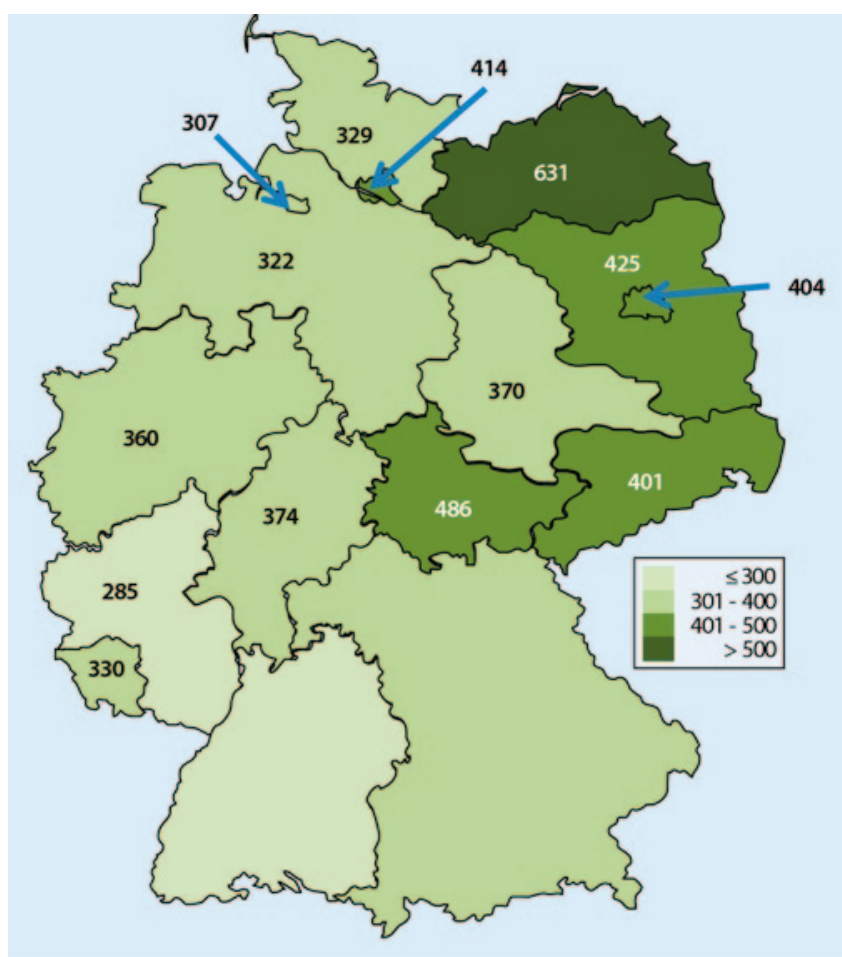

Abb. $19 \Delta$ Implantationsrate pro 1 Mio. Einwohner in den einzelnen deutschen Bundesländern (korrigiert nach Vollständigkeit, Minimaldatensätze berücksichtigt)

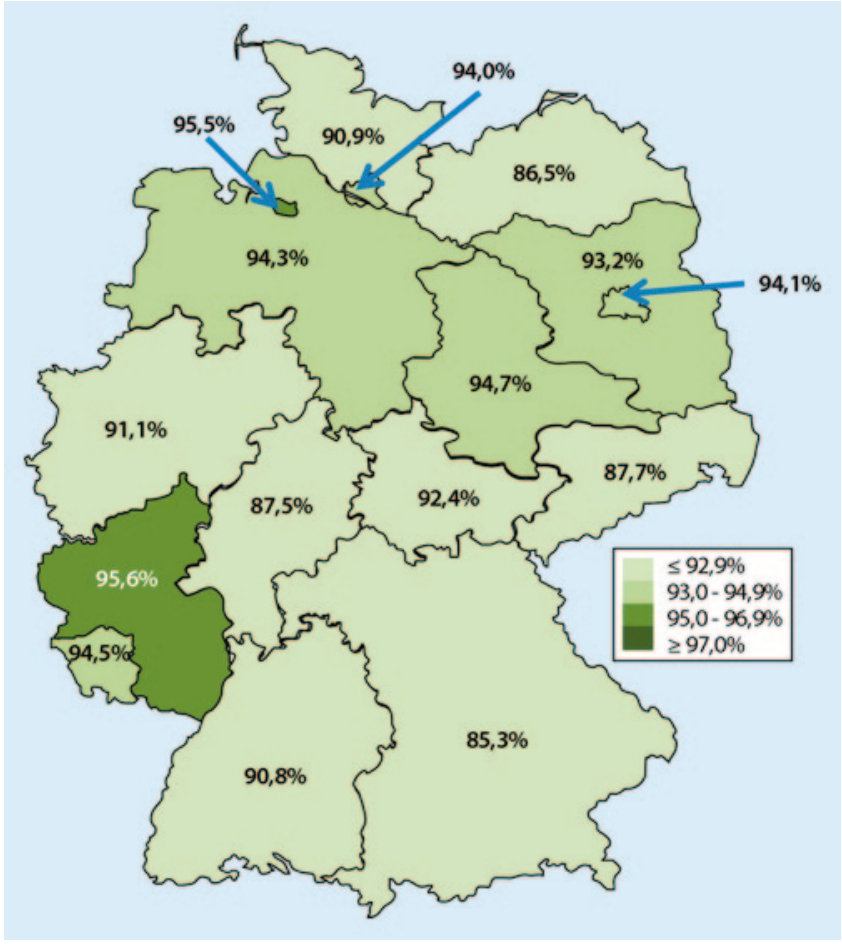

Abb. $20 \Delta$ Grad der leitlinienkonformen Indikationsstellung zur ICD-Implantation in den einzelnen deutschen Bundesländern

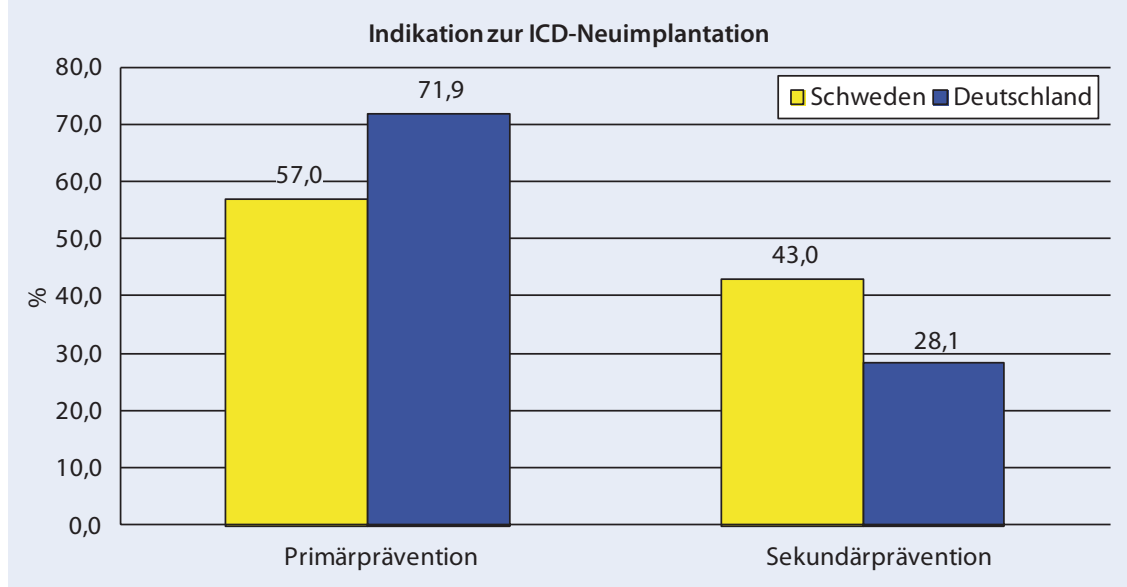

Abb. $21 \Delta$ Indikationen zur ICD-Implantation im internationalen Vergleich

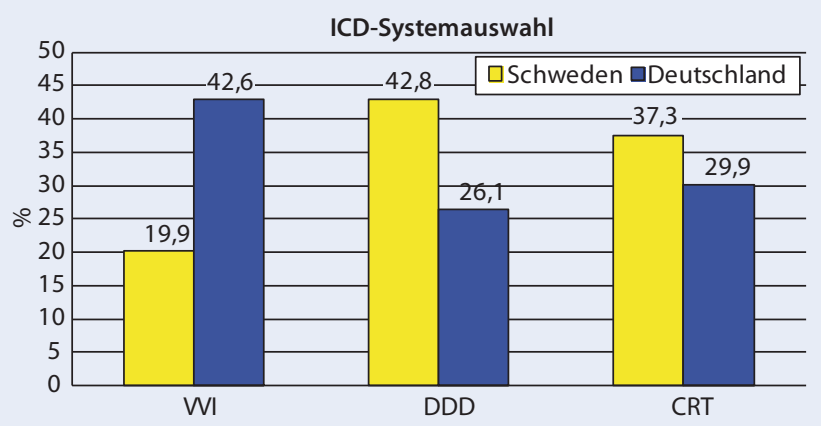

Abb. $22 \Delta$ Auswahl des ICD-Systems im Vergleich 


\section{Deutsches Herzschrittmacher- und Defibrillator-Register}

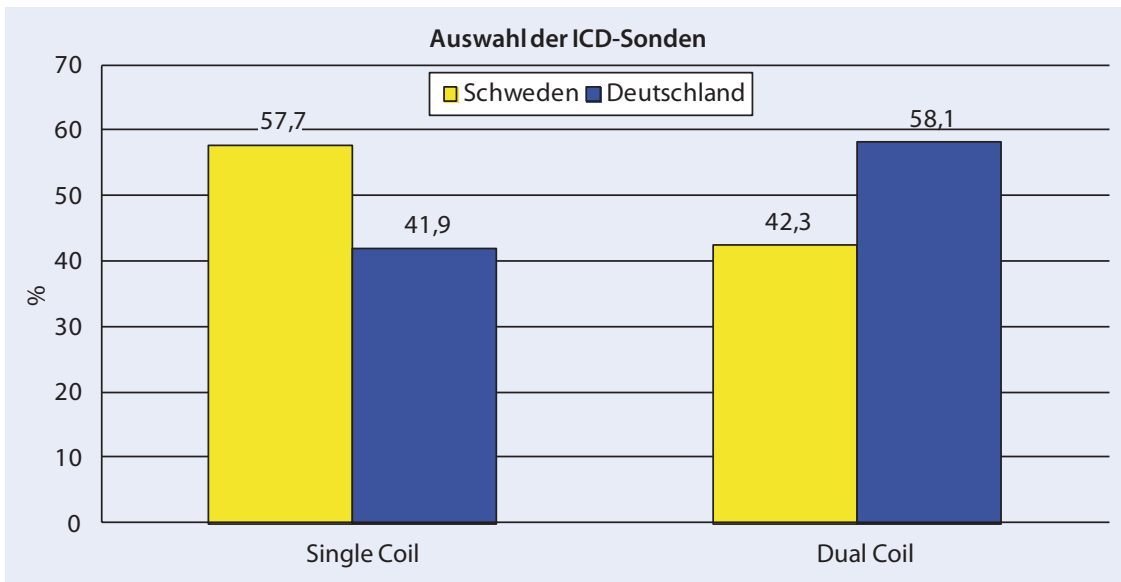

Abb. $23 \Delta$ Auswahl der ICD-Sonden im Vergleich

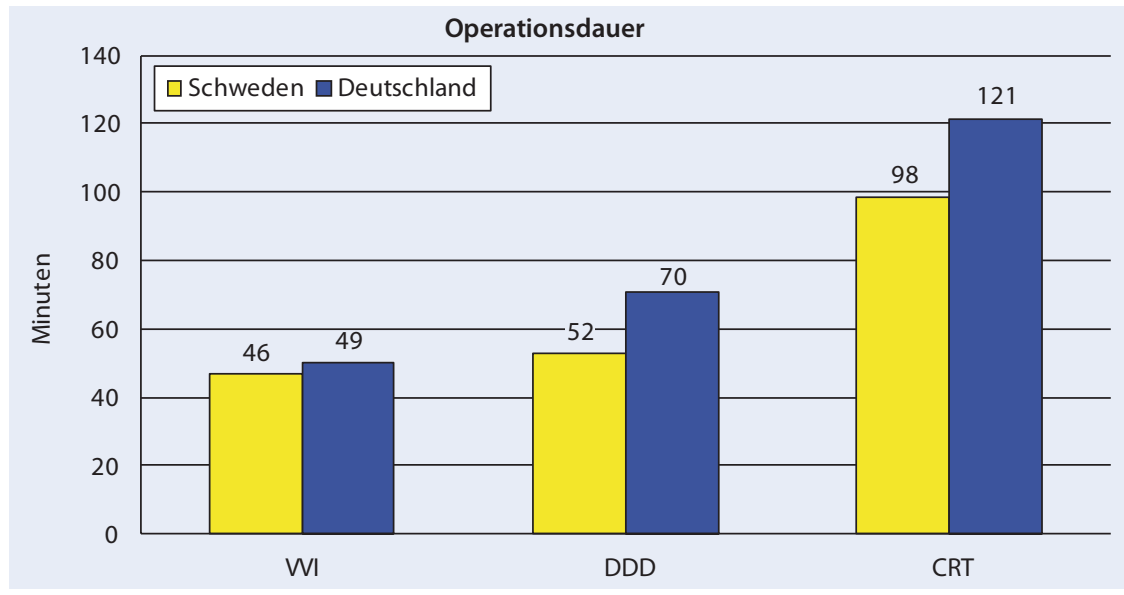

Abb. $24 \Delta$ Vergleich der mittleren Operationsdauer bei Implantationen für verschiedene Systeme

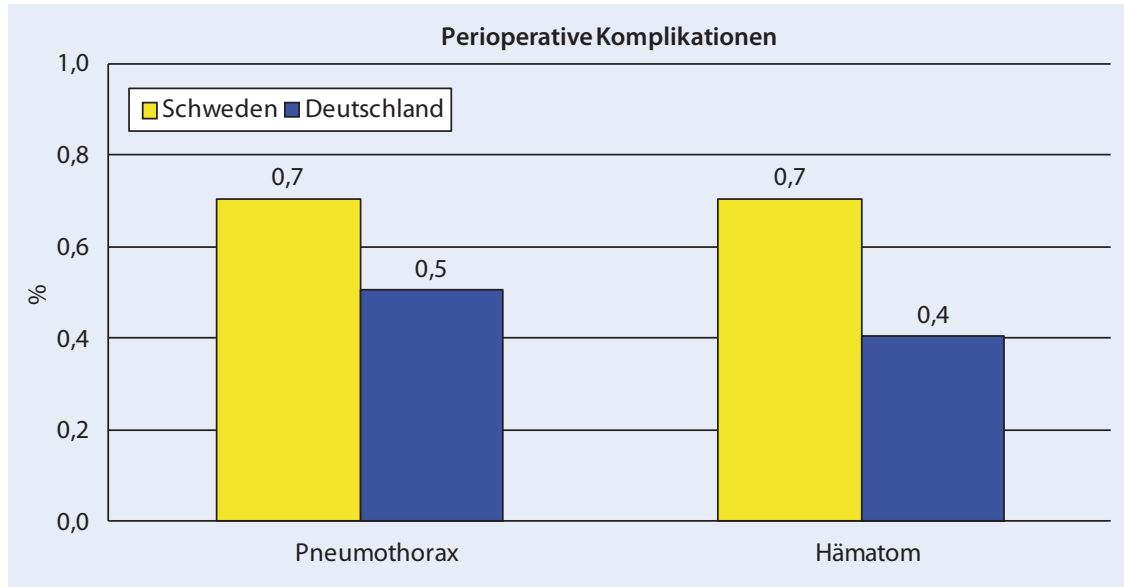

Abb. $25 \Delta$ Auftreten von perioperativen Komplikationen bei Implantationen im Vergleich 


\section{Tabellenverzeichnis}

Tabelle 1: Datenbasis 2011 im Vergleich zum Vorjahr

Tabelle 2: Operationsvolumina der meldenden Krankenhäuser

Tabelle 3: Vollzähligkeit der ausgewerteten Datensätze bzw. Krankenhäuser im Jahr 2010

(Minimaldatensätze berücksichtigt)

Tabelle 4: Vollzähligkeit der ausgewerteten Datensätze bzw. Krankenhäuser im Jahr 2011 (Minimaldatensätze berücksichtigt)

Tabelle 5: Demographische Daten zu Implantationen, Aggregatwechseln und Revisionen/Systemwechseln/Explantationen

Tabelle 6: Führende Indikation der ICD-Implantation

Tabelle 7: Verteilung der Indikation Primärprävention bei Implantationen in den einzelnen Krankenhäusern

Tabelle 8: Defibrillationselektroden und Position bei Implantationen der ersten Ventrikelsonde/Defibrillationssonde

Tabelle 9: Weitere Defibrillationssonden

Tabelle 10: Venöser Zugang bei ICD-Implantationen

Tabelle 11: Operationszeiten in Minuten bei Implantationen 2010 und 2011

(bezogen auf alle Fälle mit gültiger Angabe zur Operationsdauer > 0; MW Mittelwert, SDStandardabweichung)

Tabelle 12: Durchleuchtungszeiten in Minuten bei Implantationen 2010 und 2011 (bezogen auf alle Fälle mit gültiger Angabe zur

Durchleuchtungsdauer >0; MW Mittelwert, SD Standardabweichung)

Tabelle 13: Mittlere Operationsdauer der Systeme nach Fallzahlklassen (Anzahl an ICD-Implantationen pro Krankenhaus in 2011)

Tabelle 14: Ergebnisse der Reizschwellenmessungen und Bestimmungen der intrakardialen Signalamplituden

bei Implantationen (jeweils bezogen auf alle Fälle mit gültiger Angabe; MW Mittelwert, SD Standardabweichung)

Tabelle 15: Durchführung des intraoperativen Defibrillationstests (Implantationen)

Tabelle 16: Position der Aggregattasche

Tabelle 17: Perioperative Komplikationen bei Implantationen in den Jahren 2010 und 2011 im Vergleich

Tabelle 18: Perioperative Komplikationen in Abhängigkeit vom venösen Zugangsweg für den Sondenvorschub

Tabelle 19: Perioperative Komplikationen in Abhängigkeit vom venösen Zugangsweg für den Sondenvorschub nach Fallzahlklassen

Tabelle 20: Laufzeit der ICD-Aggregate in Jahren bezogen auf das ICD-System (Datensatz 09/5 Defibrillatoren-Aggregatwechsel, nur gültige Angaben zur Lebensdauer und zum implantierten ICD-System wurden ausgewertet)

Tabelle 21: Häufigkeit der Therapieabgabe der ausgetauschten ICD-Aggregate

Tabelle 22: Durchführung des intraoperativen Defibrillationstests (Aggregatwechsel)

Tabelle 23: Ort des letzten Eingriffs, welcher der Revisionsoperation vorausging

Tabelle 24: Indikation zur Revisionsoperation (Mehrfachnennung möglich)

Tabelle 25: Indikation zur Revisionsoperation bei Aggregatproblemen bzw. Systemumwandlungen

(Mehrfachnennungen möglich)

Tabelle 26: Indikation zur Revisionsoperation bei Sondenproblemen

Tabelle 27: Komplikationen bei Aggregatwechsel und Revisionen/Systemwechsel/Explantationen 2011 (Vorjahresdaten in Klammern; Basis der Prozentberechnung sind jeweils alle Aggregatwechsel bzw. Revisionen/Systemwechsel/Explantationen)

Tabelle 28: Todesfälle im Zusammenhang mit Implantationen, Aggregatwechseln und Revisionen/Systemwechseln/Explantationen in $2011 \mathrm{im}$ Vergleich zu den Vorjahresdaten

Tabelle 29: Datenbasis im internationalen Vergleich

Tabelle 30: Prozentuale Verteilung venöser Zugänge bei Neuimplantationen im Vergleich

Tabelle 31:Verhalten der Krankenhäuser bei Implantationen bezüglich des bevorzugten venösen Zugangs, der Auswahl der

Defibrillationselektroden sowie der Häufigkeit von Ventrikelsondendislokationen in den einzelnen Krankenhäusern im Vergleich der Jahre 2010 und 2011 


\section{Tabellenverzeichnis: Anhang 2}

Anhang 2 Tabelle 1: Operationsvolumen in Deutschland

Anhang 2 Tabelle 2: Altersverteilung der Patienten mit Implantation in Deutschland 2010 und 2011

(nur Fälle mit gültiger Altersangabe)

Anhang 2 Tabelle 3: Indikationsbegründendes klinisches Ereignis bei ICD-Implantationen in 2010 und 2011

Anhang 2 Tabelle 4: Führende klinische Symptomatik der Arrhythmie bei Implantationen (nur Patienten mit indikationsbegründendem klinischem Ereignis)

Anhang 2 Tabelle 5: Prozentuale Häufigkeit einer leitlinienkonformen Indikationsstellung bei Implantationen

Anhang 2 Tabelle 6: Details der Patienten mit einer koronaren Herzkrankheit (KHK) bei Implantationen

Anhang 2 Tabelle 7: Indikationsbegründendes klinisches Ereignis innerhalb von $48 \mathrm{~h}$ nach Infarktbeginn

(Basis der Prozentberechnung sind Patienten mit Myokardinfarkt und indikationsbegründendem klinischem Ereignis)

Anhang 2 Tabelle 8: Formen der Herzerkrankung bei Implantationen

Anhang 2 Tabelle 9: Durchführung einer medikamentösen Herzinsuffizienztherapie (zum Zeitpunkt der Diagnosestellung) bei Implantationen

Anhang 2 Tabelle 10: EKG-Befunde bei Implantationen

Anhang 2 Tabelle 11: Verteilung der mittleren Operations- und Durchleuchtungsdauer bei Implantation in den meldenden Institutionen (nur Fälle mit gültigen Angaben über 0 Min)

Anhang 2 Tabelle 12: Position der zweiten Ventrikelsonde bei Implantationen

Anhang 2 Tabelle 13: Position der dritten Ventrikelsonde bei Implantationen

Anhang 2 Tabelle 14: Ergebnisse der Reizschwellenmessungen und Bestimmungen der intrakardialen Signalamplituden für die 2. und 3. Ventrikelsonde bei Implantationen (jeweils bezogen auf alle Fälle mit gültiger Angabe; MW Mittelwert, SD Standardabweichung)

Anhang 2 Tabelle 15: Chirurgisches Vorgehen bei der Sondenrevision

Anhang 2 Tabelle 16: Durchführung des intraoperativen Defibrillationstests (Revisionen/Systemwechsel/Explantationen) Anhang 2 Tabelle 17: ICD-Implantationsrate pro 1 Mio. Einwohner aufgeteilt nach Bundesländern und adjustiert nach der Vollständigkeit der Datenerfassung 


\section{Abbildungsverzeichnis}

Abbildung 1: Prozentuale Verteilung des Operationsvolumens der einzelnen Krankenhäuser (Beispiel: Im Jahr 2011 führten 25,8\% der Einrichtungen zwischen 20 und 49 ICD-Implantationen bzw.-Aggregatwechsel durch, im Jahr 2010 waren es 25,3\%.)

Abbildung 2: Prozentuale Verteilung der Indikation Primärprävention in den einzelnen Krankenhäusern (Beispiel: Bei 4,0\% der Krankenhäuser lag der Anteil der Indikation Primärprävention zwischen 40 und $<50 \%$ in 2011)

Abbildung 3: Prozentuale Verteilung der indikationsbegründenden klinischen Ereignisse bei Sekundärprävention

Abbildung 4: Prozentuale Verteilung der ICD-Systeme bei Implantationen (Sonstiges Sonstige + VDD)

Abbildung 5: Verteilung der Häufigkeit der Verwendung der Vena cephalica bei der Implantation (Beispiel: Bei 26 Krankenhäusern lag der Anteil der Patienten mit Verwendung der Vena cephalica zwischen 20 und $<30 \%$ in 2011)

Abbildung 6: Prozentuale Verteilung der Operationszeiten bei der Implantation von Einkammersystemen (VVI) bezogen auf alle Implantationen von Einkammersystemen (VVl; Beispiel: Bei 61,2\% der implantierten Einkammersysteme lag die Operationsdauer zwischen 30 und < 60 Min)

Abbildung 7: Prozentuale Verteilung der Operationszeiten bei der Implantation von Zweikammersystemen bezogen auf alle Implantationen von Zweikammersystemen (Beispiel: Bei 41,2\% der implantierten DDD-Systeme lag die Operationsdauer zwischen 30 und < 60 Min)

Abbildung 8: Prozentuale Verteilung der Operationszeiten bei der Implantation von CRT-Systemen bezogen auf alle Implantationen von CRT-Systemen (Beispiel: Bei 6,8\% der implantierten CRT-Systeme lag die Operationsdauer zwischen 30 und $<60$ Min)

Abbildung 9: Prozentuale Verteilung der Operationsdauer bei der Implantation von Einkammersystemen (VVI) bezogen auf die Mittelwerte der Krankenhäuser (Beispiel: Bei 3,2\% aller Krankenhäuser lag der Mittelwert der Operationsdauer einer VVI-Implantation bei unter 30 Min)

Abbildung 10: Prozentuale Verteilung der Operationsdauer bei der Implantation von Zweikammersystemen bezogen auf die Mittelwerte der Krankenhäuser (Beispiel: Bei 0,2\% aller Krankenhäuser lag der Mittelwert der Operationsdauer einer DDD-Implantation bei unter < 30 Min)

Abbildung 11: Prozentuale Verteilung der Operationsdauer bei der Implantation von CRT-Systemen bezogen auf die Mittelwerte der Krankenhäuser (Beispiel: Bei 0,0\% aller Krankenhäuser lag der Mittelwert der Operationsdauer einer CRT-Implantation bei unter $<30$ Min)

Abbildung 12: Überblick über die perioperativen Komplikationen nach Implantation (Pneu interventionspflichtiger Pneumothorax; Hämatom interventionspflichtiges Taschenhämatom, Sonde A Sondendislokation der Vorhofelektrode, Sonde V Sondendislokation der Ventrikelelektrode, Infektion postoperative Wundinfektion nach Definition der CDC, Sonstige Fälle mit mindestens einer der folgenden perioperativen Komplikationen: kardiopulmonale Reanimation, interventionspflichtiger Perikarderguss, interventionspflichtiger Hämatothorax, Sondendislokation anderer Defibrillationssonden, Sondendysfunktion oder sonstige interventionspflichtige perioperative Komplikation)

Abbildung 13: Verteilung der Häufigkeit von Dislokationen der Vorhofsonde je Krankenhaus bei Implantationen (Beispiel: 545 Krankenhäuser wiesen in unter 1\% ihrer Fälle eine Vorhofsondendislokation auf; Basis der Prozentberechnung sind alle implantierten Systeme mit Vorhofsonde)

Abbildung 14: Verteilung der Aggregatlaufzeiten (Beispiel: 2011 lag bei 78,6\% der Austauscheingriffe mit Laufzeitangabe die Laufzeit zwischen 4 und $<8$ Jahren)

Abbildung 15: Chirurgisches Vorgehen bei der Sondenrevision. Bezug: alle postoperativ funktionell aktiven Sonden (1. Sonde), an denen ein Eingriff vorgenommen wurde

Abbildung 16: Chirurgisches Vorgehen bei funktionslosen Sonden. Bezug: alle postoperativ funktionell nicht aktiven Sonden (1.

Sonde), bei denen die Art des Vorgehens dokumentiert wurde

Abbildung 17: Implantationen pro 1 Mio. Einwohner im internationalen Vergleich

Abbildung 18: Anteil älterer Patienten $\geq 80$ Jahre an allen Patienten, bei denen ein ICD implantiert wurde, im Vergleich

Abbildung 19: Implantationsrate pro 1 Mio. Einwohner in den einzelnen deutschen Bundesländern (korrigiert nach Vollständigkeit, Minimaldatensätze berücksichtigt)

Abbildung 20: Grad der leitlinienkonformen Indikationsstellung zur ICD-Implantation in den einzelnen deutschen Bundesländern

Abbildung 21: Indikationen zur ICD-Implantation im internationalen Vergleich

Abbildung 22: Auswahl des ICD-Systems im Vergleich

Abbildung 23: Auswahl der ICD-Sonden im Vergleich

Abbildung 24: Vergleich der mittleren Operationsdauer bei Implantationen für verschiedene Systeme

Abbildung 25: Auftreten von perioperativen Komplikationen bei Implantationen im Vergleich 


\section{Anhang 2: Detaillierte Tabellen}

\begin{tabular}{|c|c|c|}
\hline \multicolumn{3}{|c|}{ Operationsvolumen 2011} \\
\hline & $\begin{array}{l}\text { Anzahl } \\
\text { Kranken- } \\
\text { häuser }\end{array}$ & Prozent \\
\hline \multicolumn{3}{|c|}{ Neuimplantationen } \\
\hline$n<20$ & 249 & 38,4 \\
\hline$n=20-49$ & 184 & 28,4 \\
\hline$n=50-99$ & 147 & 22,7 \\
\hline$n \geq 100$ & 68 & 10,5 \\
\hline Summe & 648 & 100,0 \\
\hline \multicolumn{3}{|c|}{ Aggregatwechsel } \\
\hline$n<20$ & 436 & 81,0 \\
\hline$n=20-49$ & 75 & 13,9 \\
\hline$n=50-99$ & 24 & 4,5 \\
\hline$n \geq 100$ & 3 & 0,6 \\
\hline Summe & 538 & 100,0 \\
\hline \multicolumn{3}{|c|}{ Revisionsoperationen } \\
\hline$n<20$ & 400 & 78,3 \\
\hline$n=20-49$ & 68 & 13,3 \\
\hline$n=50-99$ & 32 & 6,3 \\
\hline$n \geq 100$ & 11 & 2,2 \\
\hline Summe & 511 & 100,0 \\
\hline
\end{tabular}

Tab. 2 Altersverteilung der Patienten mit Implantation in Deutschland 2010 und 2011 (nur Fälle mit gültiger Altersangabe)

\begin{tabular}{lllll}
\hline Alter (Jahre) & $\mathbf{2 0 1 0}$ & & $\mathbf{2 0 1 1}$ & \\
\hline & $\boldsymbol{n}$ & Prozent & $\boldsymbol{n}$ & Prozent \\
\hline $0-9$ & 11 & $<0,1$ & 13 & $<0,1$ \\
\hline $10-19$ & 85 & 0,3 & 79 & 0,3 \\
\hline $20-29$ & 215 & 0,8 & 213 & 0,7 \\
\hline $30-39$ & 389 & 1,5 & 434 & 1,5 \\
\hline $40-49$ & 1757 & 6,9 & 1766 & 6,2 \\
\hline $50-59$ & 4277 & 16,7 & 4757 & 16,7 \\
\hline $60-69$ & 6882 & 26,9 & 7288 & 25,6 \\
\hline $70-79$ & 9850 & 38,5 & 11.312 & 39,8 \\
\hline $80-89$ & 2100 & 8,2 & 2561 & 9,0 \\
\hline$\geq 90$ & 16 & 0,1 & 29 & 0,1 \\
\hline Summe & 25.582 & 100,0 & 28.452 & 100,0 \\
\hline
\end{tabular}

\begin{tabular}{|c|c|c|c|c|}
\hline \multirow{2}{*}{$\begin{array}{l}\text { Indikationsbegründendes klini- } \\
\text { sches Ereignis }\end{array}$} & \multicolumn{2}{|l|}{2010} & \multicolumn{2}{|l|}{2011} \\
\hline & $n$ & Prozent & $n$ & Prozent \\
\hline $\begin{array}{l}\text { Indikationsbegründendes klinisches } \\
\text { Ereignis (Sekundärprävention)a }\end{array}$ & 9726 & 38,0 & 9.600 & 33,7 \\
\hline - Kammerflimmern & 2681 & 10,5 & 2.880 & 10,1 \\
\hline $\begin{array}{l}\text { - Kammertachykardie, anhaltend } \\
\text { (> } 30 \mathrm{~s} \text { ) }\end{array}$ & 2796 & 10,9 & 2.957 & 10,4 \\
\hline $\begin{array}{l}\text { - Kammertachykardie, nicht anhal- } \\
\text { tend ( } \leq 30 \text { s, aber über } 3 \text { R-R-Zyklen } \\
\text { und HF über } 100 \text { ) }\end{array}$ & 2040 & 8,0 & 2.014 & 7,1 \\
\hline - Synkope ohne EKG-Dokumentation & 1001 & 3,9 & 1.024 & 3,6 \\
\hline - Sonstiges & 1208 & 4,7 & 725 & 2,5 \\
\hline $\begin{array}{l}\text { Kein indikationsbegründendes klini- } \\
\text { sches Ereignis (Primärprävention) }\end{array}$ & 15.856 & 62,0 & 18.852 & 66,3 \\
\hline \multicolumn{5}{|c|}{ aDavon 3561 Patienten mit Myokardinfarkt (Anhang $1 \bullet$ Tab. 7) } \\
\hline
\end{tabular}

Tab. 4 Führende klinische Symptomatik der Arrhythmie bei Implantationen (nur Patienten mit indikationsbegründendem klinischem Ereignis)

\begin{tabular}{lllll}
\hline $\begin{array}{l}\text { Führende klinische Symptoma- } \\
\text { tik der Arrhythmie }\end{array}$ & $\mathbf{2 0 1 0}$ & & $\mathbf{2 0 1 1}$ & \\
\cline { 2 - 5 } & $\boldsymbol{n}$ & Prozent & $\boldsymbol{n}$ & Prozent \\
\hline Keine & 1114 & 4,4 & 926 & 3,3 \\
\hline $\begin{array}{l}\text { Herz-Kreislauf-Stillstand (reani- } \\
\text { mierter Patient) }\end{array}$ & 2854 & 11,2 & 3.126 & 11,0 \\
\hline Kardiogener Schock & 355 & 1,4 & 370 & 1,3 \\
\hline Lungenödem & 350 & 1,4 & 316 & 1,1 \\
\hline Synkope & 2285 & 8,9 & 2314 & 8,1 \\
\hline Präsynkope & 1372 & 5,4 & 1448 & 5,1 \\
\hline $\begin{array}{l}\text { Sehr niedriger Blutdruck (z. B. } \\
\text { unter 80 mmHg systolisch) }\end{array}$ & 333 & 1,3 & 414 & 1,5 \\
\hline Angina pectoris & 170 & 0,7 & 179 & 0,6 \\
\hline Sonstiges & 893 & 3,5 & 507 & 1,8 \\
\hline
\end{tabular}


Tab. 5 Prozentuale Häufigkeit einer leit-

linienkonformen Indikationsstellung bei

Implantationen

\section{Leitlinienkonforme In- Prozent dikation 2011}

Führende Indikation für ICD-Implantation

\begin{tabular}{ll}
\hline Primärprävention & 91,8 \\
\hline Sekundärprävention & 87,3 \\
\hline Summe & 90,6
\end{tabular}

Indikationsbegründendes klinisches Ereignis

\begin{tabular}{|c|c|}
\hline Kammerflimmern & 95,4 \\
\hline $\begin{array}{l}\text { Kammertachykardie, an- } \\
\text { haltend (>30 s) }\end{array}$ & 95,3 \\
\hline $\begin{array}{l}\text { Kammertachykardie, } \\
\text { nicht anhaltend ( } \leq 30 \mathrm{~s} \text {, } \\
\text { aber über } 3 \text { R-R-Zyklen } \\
\text { und HF über 100) }\end{array}$ & 65,3 \\
\hline $\begin{array}{l}\text { Synkope ohne EKG-Doku- } \\
\text { mentation }\end{array}$ & 75,2 \\
\hline Sonstiges & 75,9 \\
\hline $\begin{array}{l}\text { Kein indikationsbegrün- } \\
\text { dendes klinisches Ereig- } \\
\text { nis (Primärprävention) }\end{array}$ & 93,2 \\
\hline Summe & 90,6 \\
\hline \multicolumn{2}{|c|}{$\begin{array}{l}\text { Indikation zur kardialen Resynchronisa- } \\
\text { tionstherapie }\end{array}$} \\
\hline Nein & 88,7 \\
\hline $\mathrm{Ja}$ & 94,5 \\
\hline Summe & 90,6 \\
\hline
\end{tabular}

\begin{tabular}{|c|c|c|c|c|}
\hline KHK & 2010 & & 2011 & \\
\hline & $n$ & Prozent & $n$ & Prozent \\
\hline Ja, ohne Myokardinfarkt & 6110 & 23,9 & 6692 & 23,5 \\
\hline Ja, mit Myokardinfarkt & 9840 & 38,5 & 11.123 & 39,1 \\
\hline$-\leq 28$ Tage & 539 & 2,1 & 530 & 1,9 \\
\hline$->28$ Tage $-<40$ Tage & 325 & 1,3 & 327 & 1,1 \\
\hline -> 40 Tage & 8976 & 35,1 & 10.266 & 36,1 \\
\hline Nein & 9632 & 37,7 & 10.637 & 37,4 \\
\hline
\end{tabular}

Tab. 7 Indikationsbegründendes klinisches Ereignis innerhalb von 48 h nach Infarktbeginn (Basis der Prozentberechnung sind Patienten mit Myokardinfarkt und indikationsbegründendem klinischem Ereignis)

\begin{tabular}{lllll}
\hline $\begin{array}{l}\text { Ereignete sich das indikationsbe- } \\
\text { gründende klinische Ereignis inner- } \\
\text { halb von 48 h nach Infarktbeginn? }\end{array}$ & $\mathbf{2 0 1 0}$ & & 2011 & \\
\hline Nein & 3360 & $91,1 \%$ & 3255 & 91,4 \\
\hline Ja & 327 & 8,9 & 306 & 8,6 \\
\hline Summe & 3687 & 100,0 & 3561 & 100,0 \\
\hline
\end{tabular}

Tab. 8 Formen der Herzerkrankung bei Implantationen

\begin{tabular}{|c|c|c|c|c|}
\hline Herzerkrankung & 2010 & & 2011 & \\
\hline & $n$ & Prozent & $n$ & Prozent \\
\hline Keine Herzerkrankung & 753 & 2,9 & 618 & 2,2 \\
\hline Ischämische Kardiomyopathie & 13.643 & 53,3 & 15.489 & 54,4 \\
\hline Dilatative Kardiomyopathie DCM & 8638 & 33,8 & 9632 & 33,9 \\
\hline Hypertensive Herzerkrankung & 612 & 2,4 & 656 & 2,3 \\
\hline Erworbener Klappenfehler & 268 & 1,0 & 302 & 1,1 \\
\hline Angeborener Herzfehler & 96 & 0,4 & 81 & 0,3 \\
\hline Brugada-Syndrom & 113 & 0,4 & 112 & 0,4 \\
\hline Kurzes QT-Syndrom & 6 & $<0,1$ & 10 & $<0,1$ \\
\hline Langes QT-Syndrom & 187 & 0,7 & 198 & 0,7 \\
\hline Hypertrophe Kardiomyopathie (HCM) & 412 & 1,6 & 490 & 1,7 \\
\hline - Abnorme Blutdruckreaktion bei Belas & ung (Blu & anstieg $\leq 2$ & $\mathrm{mHg})$ & \\
\hline- Nein & 235 & 0,9 & 288 & 1,0 \\
\hline$-\mathrm{Ja}$ & 77 & 0,3 & 96 & 0,3 \\
\hline - Unbekannt & 100 & 0,4 & 106 & 0,4 \\
\hline Septumdicke $\geq 30 \mathrm{~mm}$ & & & & \\
\hline- Nein & 257 & 1,0 & 305 & 1,1 \\
\hline$-\mathrm{Ja}$ & 127 & 0,5 & 162 & 0,6 \\
\hline - Unbekannt & 28 & 0,1 & 23 & 0,1 \\
\hline $\begin{array}{l}\text { Arrhythmogene rechtsventrikuläre } \\
\text { Kardiomyopathie (ARVC) }\end{array}$ & 98 & 0,4 & 78 & 0,3 \\
\hline - Ausgeprägte rechtsventrikuläre Dysp & sie oder & ntrikuläre & iligung & \\
\hline- Nein & 38 & 0,1 & 31 & 0,1 \\
\hline$-\mathrm{Ja}$ & 52 & 0,2 & 44 & 0,2 \\
\hline - Unbekannt & 8 & $<0,1$ & 3 & $<0,1$ \\
\hline Sonstige Herzerkrankung & 756 & 3,0 & 786 & 2,8 \\
\hline
\end{tabular}




\section{Deutsches Herzschrittmacher- und Defibrillator-Register}

Tab. 9 Durchführung einer medikamentösen Herzinsuffizienztherapie (zum Zeitpunkt der

Diagnosestellung) bei Implantationen

\begin{tabular}{lllll}
$\begin{array}{lllll}\text { Medikamentöse Herzinsuffizienz- } \\
\text { therapie (zum Zeitpunkt der Diag- } \\
\text { nosestellung) durchgeführt? }\end{array}$ & $\mathbf{2 0 1 0}$ & & $\mathbf{2 0 1 1}$ & \\
\cline { 2 - 5 } Nein & 1796 & 7,0 & 1692 & 5,9 \\
\hline Ja & 23.786 & 93,0 & 26.760 & 94,1 \\
\hline - Betablocker & 22.353 & 87,4 & 25.444 & 89,4 \\
\hline - AT-Rezeptor-Blocker/ACE-Hemmer & 21.869 & 85,5 & 24.827 & 87,3 \\
\hline - Diuretika & 20.545 & 80,3 & 23.372 & 82,1 \\
\hline - Aldosteronantagonisten & 12.003 & 46,9 & 14.155 & 49,8 \\
\hline - Herzglykoside & 3883 & 15,2 & 3819 & 13,4 \\
\hline
\end{tabular}

Tab. 10 EKG-Befunde bei Implantationen

\begin{tabular}{|c|c|c|c|c|}
\hline \multirow[t]{2}{*}{ EKG-Befunde } & \multicolumn{2}{|l|}{2010} & \multicolumn{2}{|l|}{2011} \\
\hline & $n$ & Prozent & $n$ & Prozent \\
\hline \multicolumn{5}{|l|}{ Vorhofrhythmus } \\
\hline Normofrequenter Sinusrhythmus & 17.196 & 67,2 & 18.685 & 65,7 \\
\hline Sinusbradykardie/SA-Blockierungen & 2109 & 8,2 & 2586 & 9,1 \\
\hline $\begin{array}{l}\text { Paroxysmales/persistierendes Vorhofflim- } \\
\text { mern/-flattern }\end{array}$ & 2330 & 9,1 & 2607 & 9,2 \\
\hline Permanentes Vorhofflimmern & 3121 & 12,2 & 3576 & 12,6 \\
\hline $\begin{array}{l}\text { Wechsel zwischen Sinusbradykardie und } \\
\text { Vorhofflimmern (BTS) }\end{array}$ & 599 & 2,3 & 888 & 3,1 \\
\hline Sonstiges & 227 & 0,9 & 110 & 0,4 \\
\hline \multicolumn{5}{|l|}{ AV-Block } \\
\hline Keiner & 19.014 & 74,3 & 20.592 & 72,4 \\
\hline AV-Block I. Grades, Überleitung $\leq 300 \mathrm{~ms}$ & 2758 & 10,8 & 3102 & 10,9 \\
\hline AV-Block I. Grades, Überleitung > $300 \mathrm{~ms}$ & 333 & 1,3 & 447 & 1,6 \\
\hline AV-Block II. Grades, Typ Wenckebach & 213 & 0,8 & 241 & 0,8 \\
\hline AV-Block II. Grades, Typ Mobitz & 399 & 1,6 & 489 & 1,7 \\
\hline AV-Block III. Grades & 1229 & 4,8 & 1362 & 4,8 \\
\hline Nicht beurteilbar wegen Vorhofflimmerns & 1636 & 6,4 & 2219 & 7,8 \\
\hline \multicolumn{5}{|l|}{ Intraventrikuläre Leitungsstörungen } \\
\hline Keine (QRS < 120 ms) & 15.380 & 60,1 & 17.111 & 60,1 \\
\hline Rechtsschenkelblock (RSB) & 882 & 3,4 & 961 & 3,4 \\
\hline Linksanteriorer Hemiblock (LAH) + RSB & 611 & 2,4 & 605 & 2,1 \\
\hline Linksposteriorer Hemiblock (LPH) + RSB & 33 & 0,1 & 47 & 0,2 \\
\hline Linksschenkelblock, QRS 120 bis $<150 \mathrm{~ms}$ & 2856 & 11,2 & 2931 & 10,3 \\
\hline Linksschenkelblock, QRS $\geq 150 \mathrm{~ms}$ & 4764 & 18,6 & 5730 & 20,1 \\
\hline Alternierender Schenkelblock & 97 & 0,4 & 91 & 0,3 \\
\hline QRS $\geq 120$ ms ohne Differenzierung & 438 & 1,7 & 479 & 1,7 \\
\hline Sonstiges & 521 & 2,0 & 497 & 1,7 \\
\hline
\end{tabular}


Tab. 11 Verteilung der mittleren Operations- und Durchleuchtungsdauer bei Implantation in den meldenden Institutionen (nur Fälle mit gültigen Angaben über $0 \mathrm{~min}$ )

\begin{tabular}{|c|c|c|c|c|c|c|c|c|c|c|}
\hline & \multicolumn{2}{|l|}{ VVI } & \multicolumn{2}{|c|}{ VDD } & \multicolumn{2}{|l|}{ DDD } & \multicolumn{2}{|l|}{ CRT } & \multicolumn{2}{|c|}{ Sonstiges } \\
\hline & $n$ & Prozent & $n$ & Prozent & $n$ & Prozent & $n$ & Prozent & $n$ & Prozent \\
\hline \multicolumn{11}{|c|}{ Operationsdauer (min) } \\
\hline$<30$ & 20 & 3,2 & 5 & 6,3 & 1 & 0,2 & 0 & 0,0 & 0 & 0,0 \\
\hline $30-59$ & 458 & 74,1 & 49 & 61,3 & 150 & 25,8 & 6 & 1,4 & 10 & 16,7 \\
\hline $60-89$ & 134 & 21,7 & 17 & 21,3 & 314 & 54,0 & 35 & 8,0 & 16 & 26,7 \\
\hline 90-119 & 5 & 0,8 & 7 & 8,8 & 97 & 16,7 & 128 & 29,2 & 9 & 15,0 \\
\hline$\geq 120$ & 1 & 0,2 & 2 & 2,5 & 20 & 3,4 & 270 & 61,5 & 25 & 41,7 \\
\hline Summe & 618 & 100,0 & 80 & 100,0 & 582 & 100,0 & 439 & 100,0 & 60 & 100,0 \\
\hline \multicolumn{11}{|c|}{ Durchleuchtungsdauer (min) } \\
\hline$<5$ & 477 & 77,4 & 60 & 75,9 & 195 & 33,5 & 2 & 0,5 & 14 & 25,5 \\
\hline $5-9$ & 124 & 20,1 & 14 & 17,7 & 266 & 45,7 & 21 & 4,8 & 11 & 20,0 \\
\hline $10-14$ & 10 & 1,6 & 2 & 2,5 & 82 & 14,1 & 55 & 12,5 & 4 & 7,3 \\
\hline$\geq 15$ & 5 & 0,8 & 3 & 3,8 & 39 & 6,7 & 361 & 82,2 & 26 & 47,3 \\
\hline Summe & 616 & 100,0 & 79 & 100,0 & 582 & 100,0 & 439 & 100,0 & 55 & 100,0 \\
\hline
\end{tabular}

Tab. 12 Position der zweiten Ventrikelsonde bei Implantationen

\begin{tabular}{|lllll}
\hline Zweite Ventrikelsonde & $\mathbf{2 0 1 0}$ & & $\mathbf{2 0 1 1}$ & \\
\hline Position & $\boldsymbol{n}$ & Prozent & $\boldsymbol{n}$ & Prozent \\
\hline Rechtsventrikulärer Apex & 193 & 0,8 & 258 & 0,9 \\
\hline Rechtsventrikuläres Septum & 113 & 0,4 & 107 & 0,4 \\
\hline Koronarvene, anterior & 290 & 1,1 & 301 & 1,1 \\
\hline Koronarvene, lateral, posterolateral & 6238 & 24,4 & 7192 & 25,3 \\
\hline Koronarvene, posterior & 438 & 1,7 & 655 & 2,3 \\
\hline Epimyokardial linksventrikulär & 255 & 1,0 & 231 & 0,8 \\
\hline Andere & 165 & 0,6 & 219 & 0,8 \\
\hline Summe & 7692 & 30,1 & 8963 & 31,5 \\
\hline
\end{tabular}

Tab. 13 Position der dritten Ventrikelsonde bei Implantationen

\begin{tabular}{lllll}
\hline Dritte Ventrikelsonde & $\mathbf{2 0 1 0}$ & & $\mathbf{2 0 1 1}$ & \\
\hline Position & $\boldsymbol{n}$ & Prozent & $\boldsymbol{n}$ & Prozent \\
\hline Rechtsventrikulärer Apex & 5 & 0,02 & 10 & 0,04 \\
\hline Rechtsventrikuläres Septum & 2 & 0,01 & 7 & 0,02 \\
\hline Koronarvene, anterior & 1 & $<0,01$ & 13 & 0,05 \\
\hline Koronarvene, lateral, posterolateral & 10 & 0,04 & 20 & 0,07 \\
\hline Koronarvene, posterior & 0 & 0,00 & 1 & $<0,01$ \\
\hline Epimyokardial linksventrikulär & 3 & 0,01 & 0 & 0,00 \\
\hline Andere & 0 & 0,00 & 4 & 0,01 \\
\hline Summe & 21 & 0,08 & 55 & 0,19
\end{tabular}

Tab. 14 Ergebnisse der Reizschwellenmessungen und Bestimmungen der intrakardialen Signalamplituden für die 2. und 3. Ventrikelsonde bei Implantationen (jeweils bezogen auf alle Fälle mit gültiger Angabe)

\begin{tabular}{|lllll}
\hline & $n$ & MW & SD & Median \\
\hline Ventrikel (2. Sonde) & & & & \\
\hline Reizschwelle & 8762 & $1,1 \mathrm{~V}$ & $0,7 \mathrm{~V}$ & $0,9 \mathrm{~V}$ \\
\hline R-Amplitude & 8228 & $14,3 \mathrm{mV}$ & $7,6 \mathrm{mV}$ & $12,6 \mathrm{mV}$ \\
\hline Ventrikel (3. Sonde) & & & & \\
\hline Reizschwelle & 46 & $0,9 \mathrm{~V}$ & $0,4 \mathrm{~V}$ & $0,9 \mathrm{~V}$ \\
\hline R-Amplitude & 40 & $15 \mathrm{mV}$ & $9 \mathrm{mV}$ & $14 \mathrm{mV}$ \\
\hline MW Mittelwert, SD Standardabweichung & & & &
\end{tabular}


Tab. 15 Chirurgisches Vorgehen bei der Sondenrevision

\begin{tabular}{lllll} 
Operatives Vorgehen & $\mathbf{2 0 1 0}$ & & 2011 \\
& Vorhofsonde & Ventrikelsonden & Vorhofsonde & Ventrikelsonden \\
\hline Neuimplantation mit Stilllegung der alten Sonde & 156 & 1173 & 212 & 1352 \\
\hline Neuimplantation mit Entfernung der alten Sonde (Wechsel) & 469 & 1630 & 503 & 1866 \\
\hline Neuimplantation zusätzlich & 879 & 1728 & 1110 & 2029 \\
\hline Neuplatzierung & 219 & 451 & 212 & 514 \\
\hline Reparatur & 27 & 57 & 27 & 72 \\
\hline Explantation & 449 & 750 & 560 & 913 \\
\hline Stillegung & 56 & 442 & 67 & 413 \\
\hline Sonstiges & 46 & 136 & 64 & 157 \\
\hline Summe & 2301 & 6367 & 2755 & 7316
\end{tabular}

Tab. 16 Durchführung des intraoperativen Defibrillationstests (Revisionen/Systemwechsel/Explantationen)

Intraoperativer Defibrillationstest bei Revisionen/Systemwechsel/Explantationen durchgeführt?

Ja

- Sicherheitsabstand: nein

- Sicherheitsabstand: ja

Nein, wegen intrakardialen Thromben

Nein, wegen hämodynamischer Instabilität (katecholaminpflichtig 270

oder Lungenödem)

Nein, aus sonstigen Gründen

3350

\section{0}

$n$

3122

91

3031

272

270

\begin{tabular}{ll} 
& 2011 \\
Prozent & $\boldsymbol{n}$ \\
\hline 44,5 & 3099 \\
\hline 1,3 & 101 \\
43,2 & 29 \\
\hline 3,9 & 336 \\
3,8 & 307
\end{tabular}

2011

Prozent

$3099 \quad 38,5$

$101 \quad 1,3$

$2998 \quad 37,2$

$336 \quad 4,2$

$307 \quad 3,8$

4314

53,6

Tab. 17 ICD-Implantationsrate pro 1 Mio. Einwohner aufgeteilt nach Bundesländern und adjustiert nach der Vollständigkeit der Datenerfassung

\begin{tabular}{|c|c|c|c|c|c|}
\hline Bundesland & $\begin{array}{l}\text { ICD-Implantationen } \\
2011\end{array}$ & $\begin{array}{l}\text { Einwohner } \\
\text { (Stand: } 31.12 .2011 \text { ) }\end{array}$ & $\begin{array}{l}\text { Implantationsrate } \\
\text { pro } 1 \text { Mio. }\end{array}$ & $\begin{array}{l}\text { Vollständigkeit } \\
\text { (\%) }\end{array}$ & $\begin{array}{l}\text { Implantationsrate pro } \\
1 \text { Mio. adjustiert nach } \\
\text { Vollständigkeit }\end{array}$ \\
\hline Baden-Württemberg & 3223 & 10.786 .227 & 299 & 100,19 & 300 \\
\hline Bayern & 3929 & 12.595 .891 & 312 & 98,13 & 318 \\
\hline Berlin & 1395 & 3.501 .872 & 398 & 98,66 & 404 \\
\hline Brandenburg & 1033 & 2.495 .635 & 414 & 97,45 & 425 \\
\hline Bremen & 202 & 661.301 & 305 & 99,51 & 307 \\
\hline Hamburg & 733 & 1.798 .836 & 407 & 98,92 & 414 \\
\hline Hessen & 2268 & 6.092 .126 & 372 & 99,78 & 374 \\
\hline Mecklenburg-Vorpommern & 1013 & 1.634 .734 & 620 & 98,25 & 631 \\
\hline Niedersachsen & 2530 & 7.913 .502 & 320 & 99,26 & 322 \\
\hline Nordrhein-Westfalen & 6308 & 17.841 .956 & 354 & 98,24 & 360 \\
\hline Rheinland-Pfalz & 998 & 3.999 .117 & 250 & 87,70 & 285 \\
\hline Saarland & 328 & 1.013 .352 & 324 & 98,80 & 330 \\
\hline Sachsen & 1632 & 4.137 .051 & 394 & 98,37 & 401 \\
\hline Sachsen-Anhalt & 842 & 2.313 .280 & 364 & 98,36 & 370 \\
\hline Schleswig-Holstein & 926 & 2.837 .641 & 326 & 99,04 & 329 \\
\hline Thüringen & 1092 & 2.221 .222 & 492 & 101,20 & 486 \\
\hline Summe & 28.452 & 81.843 .743 & 348 & 98,33 & 354 \\
\hline $\begin{array}{l}\text { Früheres Bundesgebiet (ohne } \\
\text { Berlin) }\end{array}$ & 21.445 & 65.539 .949 & 327 & 98,32 & 333 \\
\hline Neue Länder (mit Berlin) & 7007 & 16.303 .794 & 430 & 98,70 & 436 \\
\hline
\end{tabular}

